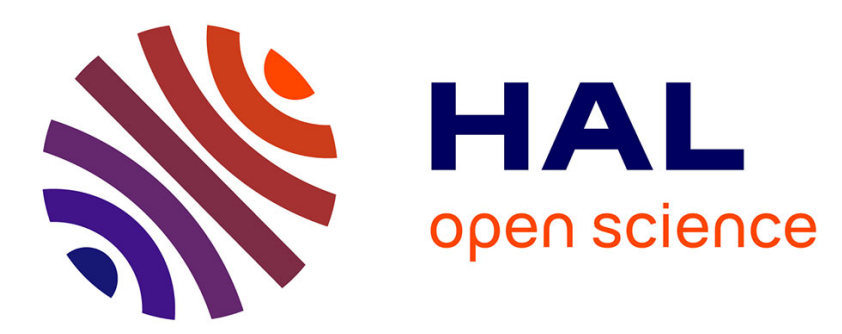

\title{
A domain coupling method for finite element digital image correlation with mechanical regularization: Application to multiscale measurements and parallel computing
}

Robin Bouclier, Jean-Charles Passieux

\section{To cite this version:}

Robin Bouclier, Jean-Charles Passieux. A domain coupling method for finite element digital image correlation with mechanical regularization: Application to multiscale measurements and parallel computing. International Journal for Numerical Methods in Engineering, 2017, 111 (2), pp.123 - 143. 10.1002/nme.5456 . hal-01577929

\section{HAL Id: hal-01577929 \\ https://hal.science/hal-01577929}

Submitted on 7 Jun 2018

HAL is a multi-disciplinary open access archive for the deposit and dissemination of scientific research documents, whether they are published or not. The documents may come from teaching and research institutions in France or abroad, or from public or private research centers.
L'archive ouverte pluridisciplinaire HAL, est destinée au dépôt et à la diffusion de documents scientifiques de niveau recherche, publiés ou non, émanant des établissements d'enseignement et de recherche français ou étrangers, des laboratoires publics ou privés. 


\title{
A domain coupling method for finite element digital image correlation with mechanical regularization: Application to multiscale measurements and parallel computing
}

\author{
Robin Bouclier ${ }^{1, *}$ and Jean-Charles Passieux ${ }^{2}$ \\ ${ }^{1}$ Université de Toulouse, INSA-Toulouse, IMT UMR CNRS 5219, 135 avenue de Rangueil, F-31077 Toulouse Cedex 04, France \\ ${ }^{2}$ Institut Clément Ader (ICA), Université de Toulouse, UMR CNRS 5312-INSA, Toulouse, France
}

\begin{abstract}
SUMMARY
A general method is proposed to couple two sub-regions analysed with finite element digital image correlation (FE-DIC) even when using a mechanical regularization (R-DIC). A Lagrange multiplier is introduced to stitch both displacements fields in order to recover continuity over the full region of interest. Another interface unknown is introduced to ensure, additionally, the equilibrium of the mechanical models used for regularization. As a first application, the method is used to perform a single measurement from images at two different resolutions. Secondly, the method is also extended to parallel computing in R-DIC. The problem is formulated at the interface and solved with GMRes. A dedicated preconditioner is proposed to significantly accelerate convergence. The resulting method is a good candidate for the analysis of large data-sets. Draft
\end{abstract}

Received ...

KEY WORDS: digital image correlation; finite elements; mechanical regularization; domain decomposition; multiscale; high-performance computing

\section{INTRODUCTION}

Digital Image correlation is nowdays one the most used full-field measurement technique for the analysis of the behavior of materials and structures. This is certainly due to the fact that it is non-contact; relatively easy to use; it makes use of multipurpose reusable hardware; its ablility to measure 2D or 3D displacement fields at the surface or in the bulk, from static to transient dynamic analyses and finally, this measurement technique is not restricted to one specific scale or resolution. Among the different families of DIC methods, the one based on finite elements (FEDIC [1]) are subject to a growing interest in the solid mechanics community [1-8]. Using FE-DIC it becomes very convenient to couple measurements with simulation software as they both use finite elements for the interpolation of the kinematic fields.

DIC is ill-posed in Hadamar's sense. Namely, it is not possible to solve this problem pointwise without considering some regularisation (with an associated characteristic length). In practice, this regularization length is associated to the window or element size when using subset or FE-DIC respectively. This way of regularizing DIC leads to a tradeoff between FE interpolation error and so-called ultimate random error [6,9]. Another great interest of using finite elements in DIC is that it is possible to regularize the DIC problem more softly, based on the knowledge of a FE mechanical model [10-16]. It can be seen as a mechanical low pass filter whose cutoff wavelenght plays the role of the above regularization length [13]. This mechanically regularised DIC (R-DIC) is of special interest when facing some challenging situations, like, for instance, in Digital Volume Correlation [13], where the speckle pattern is not rich enough [17] or too much anisotropic [18]. Finally, R-DIC allows also to devise very efficient identification strategies [11, 14].

${ }^{*}$ Correspondence to: Robin Bouclier, Université de Toulouse, INSA-Toulouse, IMT UMR CNRS 5219, 135 avenue de Rangueil, F-31077 Toulouse Cedex 04, France.

†E-mail: bouclier@insa-toulouse.fr

draft

Prepared using nmeauth.cls [Version: 2010/05/13 v3.00] 
In this paper, a way to couple different domains analysed by R-DIC is presented. The challenge is that not only the displacement must be continuous across the interface, but also the underlying mechanical models used to regularize must be in equilibrium. If the kinematic compatibility can be classically enforced by introducing a Lagrange multiplier, the static constraint requires a specific treatment. Indeed, while in the field of computational mechanics the minimization of the potential energy is generally encountered, the mechanical regularization in RDIC relies on the minimization of the $L^{2}$-norm of the internal forces. To be able to split the regularization term, we propose to make use of a new interface unknown. This additional variable can be interpreted as the interface reaction force but does not constitute a Lagrange multiplier from a numerical point of view. This leads to the construction of an original mixed formulation that is suitable for the accurate resolution of the correlation problem. We believe that the developed method is of importance in the field of DIC. Indeed, it could be useful in the following situations:

- multiscale or multi-resolution DIC: when at least two DIC measurements are done with different resolutions on the same experiment $[6,19]$. For instance, it was shown that such a multiscale measurement makes it is possible to reduce significantly the identification uncertainties [6]. Bonding the displacements fields comming from different scales seems of particular interest, especially in the context of FE-DIC where the displacement continuity is physically relevant.

- multiscale regularisation model: when the mechanical model used for the regularization is multiscale. There are many engineering situations where the model is the result of a global coarse model locally enhanced by a more complex, but smaller, model [20-22]. For instance, in [15], two different mechanical models are used in the same measurement, a Williams' series expansion close to the crack tip and a more classical FE elastic model in the remainder of the domain.

- high performance computing: when large data sets are used (high resolution images, DVC...), the price to pay is that FE-DIC, like any DIC method formulated globally in the whole region of interest (global DIC), lead to the resolution of a large system of equations. In opposition more classical subset based methods result in a large set a small problems which is by nature directly parallelisable. Recently, a parallel FE-DIC solver was proposed in [23] which is based on a so-called domain decomposition solver that were initially developed for computational mechanics. The method considers a non-overlapping partition of the mesh into a set of subdomains, along with an overlapping partition of the images into a set of subset images (which is classical to subset based methods). The method proved to be highly parallelisable. It can be seen as a bridge between subset and FE DIC methods.

After a brief presentation of FE-DIC and R-DIC in section 2, the proposed coupling method is presented in section 3. The kinematic coupling is ensured by introducing a Lagrange multiplier on the interface and another interface variable is introduced to perform the static coupling (equilibrium). In sections 4 and 5, two applications of the method are developed to assess the performance of the proposed coupling. First, the method is used in section 4 to measure a multiscale regular and continuous displacement field on a specimen taking the best of a set of farfield and nearfield images. Then, the method is extended in section 5 to parallel computing. The coupled problem is condensed on the interface and solved using a Krylov type iterative solver. A preconditioner is also proposed to help reduce the computational cost of the method.

\section{THE REFERENCE DIGITAL IMAGE CORRELATION PROBLEM}

This section establishes the context of the study and introduces the corresponding notations. A brief review of DIC is given with a particular emphasis on the concept of mechanical regularization. For further details, the interested reader is referred to the references cited hereafter.

\subsection{Optical flow}

Let us consider two grayscale images $f(\mathbf{x})$ and after $g(\mathbf{x})$ corresponding to the reference and deformed states of the specimen respectively. The displacement field between those two states is denoted $\mathbf{u}(\mathbf{x})$, where $\mathbf{x} \in \Omega$ gives the position of a pixel in the region of interest $\Omega$ of the image. The optical flow equation [24] states that the gray level variation between the two images is due to only the passive advection of image texture:

$$
f(\mathbf{x})=g(\mathbf{x}+\mathbf{u}(\mathbf{x})) .
$$

draft

Prepared using nmeauth.cls

Int. J. Numer. Meth. Engng (2016)

DOI: $10.1002 / \mathrm{nme}$ 
Equation (1) is solved in a least square sense. The resulting problem can be written in the continuous setting as follows: find $\mathbf{u} \in \mathbf{L}^{2}(\Omega)$ (where $\mathbf{L}^{2}(\Omega)$ defines Hilbert space $\left[L^{2}(\Omega)\right]^{d}$ with $d=2$ for DIC and 3 for DVC) that minimizes the quadratic distance $\Phi_{\text {cor }}(\mathbf{u})$ :

$$
\Phi_{c o r}(\mathbf{u})=\frac{1}{2} \int_{\Omega}(f(\mathbf{x})-g(\mathbf{x}+\mathbf{u}(\mathbf{x})))^{2} d \mathbf{x}
$$

Problem (2) is nonlinear. Assuming that $g$ (and thus $f$ ) is differentiable, the minimization of the cost function (2) can be performed using a Gauss-Newton algorithm. At each iteration $k$, an estimate of $\mathbf{u}^{k-1}$ being known, the unknown displacement correction $\delta \mathbf{u}^{k}=\mathbf{u}^{k}-\mathbf{u}^{k-1}$ is searched for as the solution of the following variational formulation [5]: find $\delta \mathbf{u}^{k} \in \mathbf{L}^{2}(\Omega)$ such that:

$$
a\left(\delta \mathbf{u}^{k}, \mathbf{v}\right)=l^{k}(\mathbf{v}) \quad \forall \mathbf{v} \in \mathbf{L}^{2}(\Omega),
$$

where:

$\tilde{a}(\mathbf{u}, \mathbf{v})=\int_{\Omega} \mathbf{v}^{T} \nabla g\left(\mathbf{x}+\mathbf{u}^{k-1}\right) \nabla g\left(\mathbf{x}+\mathbf{u}^{k-1}\right)^{T} \mathbf{u} d \mathbf{x} ; \tilde{l}^{k}(\mathbf{v})=\int_{\Omega} \mathbf{v}^{T} \nabla g\left(\mathbf{x}+\mathbf{u}^{k-1}\right)\left(f(\mathbf{x})-g\left(\mathbf{x}+\mathbf{u}^{k-1}\right)\right) d \mathbf{x}$.

In equation (4), $\nabla g\left(\mathbf{x}+\mathbf{u}^{k-1}\right)$ denotes the gradient of image $g$ at non integer pixels positions $\mathbf{x}+\mathbf{u}^{k-1}$. In practice, since $g\left(\mathbf{x}+\mathbf{u}^{k-1}\right)$ is supposed to converge to $f(\mathbf{x})$, the gradient $\nabla g\left(\mathbf{x}+\mathbf{u}^{k-1}\right)$ is approximated by $\nabla f(\mathbf{x})[25,26]$, which does not depend on the unknown and which can then be computed once and for all. We thus end up with the following modified forms to be considered in formulation (3):

$$
a(\mathbf{u}, \mathbf{v})=\int_{\Omega} \mathbf{v}^{T} \nabla f \nabla f^{T} \mathbf{u} d \mathbf{x} ; l^{k}(\mathbf{v})=\int_{\Omega} \mathbf{v}^{T} \nabla f\left(f(\mathbf{x})-g\left(\mathbf{x}+\mathbf{u}^{k-1}\right)\right) d \mathbf{x} .
$$

As such, this problem is ill-posed in Hadamar's sense; that is, the displacement cannot be found pixel-wise. As a consequence, the unknown displacement $\delta \mathbf{u}^{k}$ is searched for in an approximation subspace $\mathcal{V} \subset \mathbf{L}^{2}(\Omega)$ generally spanned by a set of basis functions $\mathbf{N}_{i}(\mathbf{x})$, such that $\delta \mathbf{u}^{k}(\mathbf{x})=\sum_{i} \mathbf{N}_{i}(\mathbf{x}) \delta q_{i}^{k}$ where $\delta q_{i}^{k}$ are the corresponding coefficients of the linear combination, referred to as degrees of freedom (d.o.f.). By applying the Galerkin method, problem (3) yields the following linear system of equations:

$$
\mathbf{M} \delta \mathbf{q}^{k}=\mathbf{b}^{k},
$$

where $\delta \mathbf{q}^{k}$ is a vector collecting the d.o.f. $\delta q_{i}^{k}$ and:

$$
\mathbf{M}_{i j}=a\left(\mathbf{N}_{i}(\mathbf{x}), \mathbf{N}_{j}(\mathbf{x})\right) ; \mathbf{b}_{j}^{k}=l^{k}\left(\mathbf{N}_{j}(\mathbf{x})\right) .
$$

The correlation operator $\mathbf{M}$ is symmetric positive definite (provided the images possess a regular texture, i.e., the gradient may vanish exclusively over a null measure subset [5]). For the choice of the basis functions $\mathbf{N}_{i}(\mathbf{x})$, finite element interpolations seem to have gathered considerable momentum in continuum solid mechanics. This leads to the finite element based DIC (FE-DIC) approach or global DIC approach (see [1-8] to name a few). In contrast to the so-called subset methods [27-29] that rely on non-continuous piecewise polynomial functions, FE-DIC allows for interpolation-free communications with finite element simulations. Nevertheless, its drawback over subset-DIC is the computational cost when high resolution is required [12,30,31]. Indeed, subset approaches involve a block diagonal correlation operator $\mathbf{M}$ whose resolution is highly parallelisable.

\subsection{Mechanical regularization}

To further reduce the measurement uncertainty in challenging situations, a mechanical regularization of the optical flow can be adopted. It consists in complementing DIC with an additional penalization on the distance between the estimated displacement field and its projection onto the space of mechanical solutions. Originally introduced for fracture analysis [10], the method may also appear necessary when dealing with poor textures (e.g., when dealing with tomographic images) or for identification purposes for instance (see, e.g., [11, 14]).

From here on, we denote $\mathbf{q}$ the vector that collects the d.o.f. $q_{i}$ of the FE displacement solution $\mathbf{u} \in \mathcal{V}$. In the context of linear elasticity, the method consists in augmenting Eq. (2) with the $L^{2}$-norm of the internal forces (in 


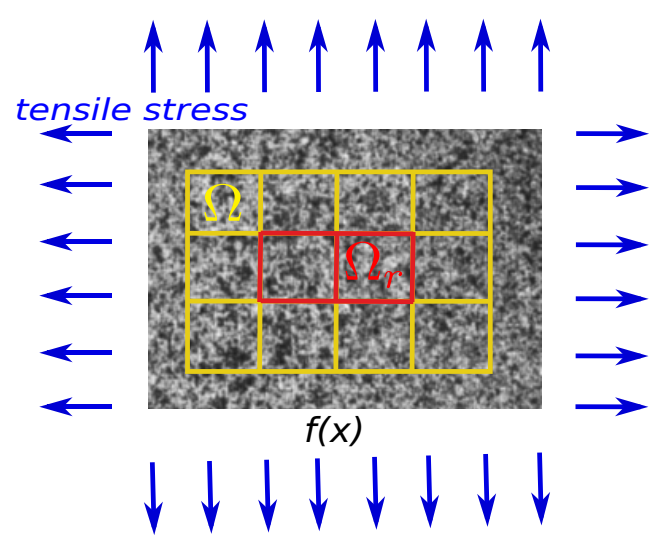

Figure 1. Reference image $f$ with its underlying mesh for FE-DIC. Note that only the d.o.f in domain $\Omega_{r}$ are regularized.

the spirit of the Equilibrium Gap Method [32]):

$$
\Phi_{r e g}(\mathbf{u})=\frac{1}{2}\|\overline{\mathbf{K}} \mathbf{q}\|_{2}^{2}=\frac{1}{2} \mathbf{q}^{T} \overline{\mathbf{K}}^{T} \overline{\mathbf{K}} \mathbf{q} .
$$

$\overline{\mathbf{K}} \mathbf{q}$ is the vector that collects the internal forces for each d.o.f. except those supported by the nodes that are concerned with Dirichlet or non-zero Neumann boundary conditions (see Fig. 1 for an illustration in the case of a bi-axial tensile test). Such a d.o.f. selection appears necessary here since we do not know well the boundary conditions. Operator $\overline{\mathbf{K}}$ is in practice obtained as follows:

$$
\overline{\mathbf{K}}=\mathbf{P} \mathbf{K},
$$

where $\mathbf{K}$ is the elastic FE stiffness matrix for the considered mesh and $\mathbf{P}$ is a selection matrix that allows to select the d.o.f. to be regularized. $\mathbf{P}$ formally reads as a diagonal matrix with one if the corresponding d.o.f. is controlled and zero otherwise. A weighted sum of both quadratic distances $\left(\Phi_{c o r}(\mathbf{u})\right.$ and $\left.\Phi_{r e g}(\mathbf{u})\right)$ is then considered for the minimization:

$$
\Phi_{\text {tot }}(\mathbf{u})=\Phi_{\text {cor }}(\mathbf{u})+\eta\left(l_{c}\right) \Phi_{\text {reg }}(\mathbf{u}),
$$

where $\eta\left(l_{c}\right)$ is the weighting parameter. It has been shown that such a regularization introduces a mechanical low-pass filter whose cut-off wavelength $l_{c}$ can be controlled [13]. It has to be noted that the elastic behavior is not prescribed in a strong way. It is only used has a low pass filter to alleviate noise effects. The low frequency part of the solution is not affected. According to us, weakly prescribing a vague elastic behavior makes sense in continuum mechanics, even if we miss information on the material properties. For the sake of readability, we omit the dependence on $l_{c}$ in the following: we write $\eta\left(l_{c}\right)=\eta$. The study and proper choice of this parameter (see, for instance, $[10,12,32]$ ) is beyond the scope of this work, whose purpose is to develop a coupling method for FE-DIC that uses such a regularization. Replacing Eq. (2) by Eq. (10) and applying the same strategy as in section 2.1, we finally obtain the following linear system to be solved to compute the Gauss-Newton update at iteration $k$ :

$$
\left(\mathbf{M}+\eta \overline{\mathbf{K}}^{T} \overline{\mathbf{K}}\right) \delta \mathbf{q}^{k}=\mathbf{b}^{k}-\eta \overline{\mathbf{K}}^{T} \overline{\mathbf{K}} \mathbf{q}^{k-1}
$$

where $\mathbf{q}^{k-1}$ denotes the d.o.f. vector corresponding to $\mathbf{u}^{k-1}$. With the values considered for $\eta$ in our simulations, the new correlation operator $\mathbf{M}+\eta \overline{\mathbf{K}}^{T} \overline{\mathbf{K}}$ appeared symmetric positive definite as its classical counterpart. In the following, this mechanically regularized DIC method is denoted by R-DIC.

\section{Remark 1}

Even if we restrict ourselves in this work to linear elastic regularization, one may note that the more general case of non-linear constitutive models, such as elasto-plasticity, may also be treated by applying the extension provided in [14]. 


\section{THE PROPOSED COUPLING METHOD}

We now address the coupling of multiple domains in R-DIC. After introducing the coupling problem to be solved, the proposed coupling formulation is constructed. It constitutes an extension of the coupling method of previous work [23] to be able to take into account an elastic regularization of the optical flow.

\subsection{Coupling problem}

Let us consider a non-overlapping partition of the region of interest $\Omega$ into a set of subdomains $\Omega_{s}$. In addition, we subdivide images $f$ and $g$ in a set of rectangular subset images $f_{s}$ and $g_{s}$ surrounding domain $\Omega_{s}$ as it is usually performed in subset-based approaches [29]. In the following, any quantity with subscript $\square_{s}$ defines the restriction to $\Omega_{s}$ of quantity $\square$. For the sake of simplicity, the method will be first presented in the case of two subdomains: $\Omega_{1}$ and $\Omega_{2}$ that are connected along interface $\Gamma$. As well, we introduce for the mechanical regularization the two subdomains $\Omega_{r_{1}}$ and $\Omega_{r_{2}}$ with their common interface $\Gamma_{r}$ (see Fig. 2, the corresponding initial FE-DIC problem being the one illustrated in Fig. 1). Regarding the discretization, even if the method applies in more general context, we consider in this work the case of matching meshes; that is, the meshes of the two subdomains along the interface are perfectly aligned.

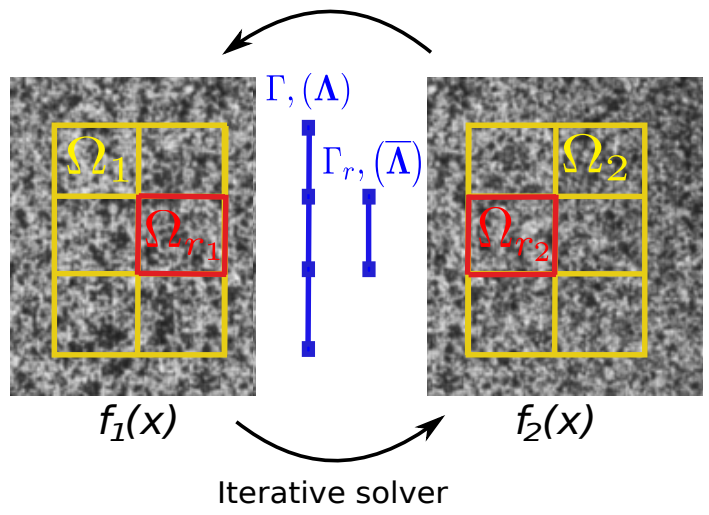

Figure 2. Domain $\Omega$ decomposition in two subdomains $\Omega_{s}$ and two subset images $f_{s}$. Note that subdomains and submeshes are not overlapping but subset images are.

\subsection{Formulation of the coupling method}

For the presentation of the method in this section, we consider only two independent R-DIC measurements, i.e., that only two sets of images $\left(f_{1}, f_{2}\right)$ and $\left(g_{1}, g_{2}\right)$ are considered in the subdivision (see Fig. 2). We are interested in this work in developing a non-overlapping coupling method; that is, we need to write the reference problem (minimization of cost function (10)) as a sum of local problems over $\Omega_{1}$ and $\Omega_{2}$ and to restrict the exchange of data to interface $\Gamma$.

3.2.1. Case of FE-DIC without mechanical regularization. When restricted to FE-DIC without mechanical regularization (minimization of cost function (2)), it suffices to make use of the additivity of the integral with respect to domain $\Omega$ in Eq. (2), and to prescribe the displacement continuity across interface $\Gamma$ to satisfy the $C^{0}$ regularity of the FE space $\mathcal{V}$. This strategy is performed in [23] by introducing (as classically in numerical coupling methods) a Lagrange multiplier $\lambda \in \mathcal{L}(\mathcal{L}$ being spanned by the trace on $\Gamma$ of the FE shape functions of $\mathcal{V})$. We obtain the following coupling formulation that is equivalent to the inital FE-DIC formulation without mechanical regularization: find $\left(\mathbf{u}_{1}, \mathbf{u}_{2}, \boldsymbol{\lambda}\right) \in \mathcal{V}_{1} \times \mathcal{V}_{2} \times \mathcal{L}$ that minimize:

$$
\frac{1}{2} \int_{\Omega_{1}}\left(f_{1}(\mathbf{x})-g_{1}\left(\mathbf{x}+\mathbf{u}_{1}(\mathbf{x})\right)\right)^{2} d \mathbf{x}+\frac{1}{2} \int_{\Omega_{2}}\left(f_{2}(\mathbf{x})-g_{2}\left(\mathbf{x}+\mathbf{u}_{2}(\mathbf{x})\right)\right)^{2} d \mathbf{x}+\int_{\Gamma} \boldsymbol{\lambda} \cdot\left(\mathbf{u}_{1}-\mathbf{u}_{2}\right) \mathrm{d} \Gamma .
$$

The third term of this equation is computed as follows:

$$
\int_{\Gamma} \boldsymbol{\lambda} \cdot\left(\mathbf{u}_{1}-\mathbf{u}_{2}\right) \mathrm{d} \Gamma=\boldsymbol{\Lambda}^{T}\left(\mathbf{C}_{1} \mathbf{q}_{1}+\mathbf{C}_{2} \mathbf{q}_{2}\right),
$$

draft

Prepared using nmeauth.cls 
where $\boldsymbol{\Lambda}$ is the d.o.f. vector corresponding to $\boldsymbol{\lambda}$ and $\mathbf{C}_{s}$ are the coupling operators. $\mathbf{C}_{s}$ formally read as rectangular signed Boolean operators such that if a d.o.f. belongs to $\Gamma$, its value is set to 1 , and the corresponding d.o.f. on the other side of $\Gamma$ is set to -1 . By applying the same strategy as in section 2.1, we obtain the coupling three-field formulation to be solved to compute the Gauss-Newton update at iteration $k: \forall\left(\mathbf{v}_{1}, \mathbf{v}_{2}, \boldsymbol{\mu}\right) \in \mathcal{V}_{1} \times \mathcal{V}_{2} \times \mathcal{L}$,

$$
\begin{aligned}
& a_{1}\left(\delta \mathbf{u}_{1}^{k}, \mathbf{v}_{1}\right)+a_{2}\left(\delta \mathbf{u}_{2}^{k}, \mathbf{v}_{2}\right)+\int_{\Gamma} \boldsymbol{\mu} \cdot\left(\delta \mathbf{u}_{1}^{k}-\delta \mathbf{u}_{2}^{k}\right) \Gamma+\int_{\Gamma} \boldsymbol{\lambda} \cdot\left(\mathbf{v}_{1}-\mathbf{v}_{2}\right) \mathrm{d} \Gamma \\
& =l_{1}^{k}\left(\mathbf{v}_{1}\right)+l_{2}^{k}\left(\mathbf{v}_{2}\right)-\int_{\Gamma} \boldsymbol{\mu} \cdot\left(\mathbf{u}_{1}^{k-1}-\mathbf{u}_{2}^{k-1}\right) \Gamma
\end{aligned}
$$

where $\boldsymbol{\mu}$ denotes the virtual Lagrange multiplier field associated to $\lambda$ and forms $a$ and $l^{k}$ read as in Eq. (5). In the discrete setting, Eq. (14) becomes:

$$
\left[\begin{array}{ccc}
\mathbf{M}_{1} & 0 & \mathbf{C}_{1}^{T} \\
0 & \mathbf{M}_{2} & \mathbf{C}_{2}^{T} \\
\mathbf{C}_{1} & \mathbf{C}_{2} & 0
\end{array}\right]\left[\begin{array}{c}
\delta \mathbf{q}_{1}^{k} \\
\delta \mathbf{q}_{2}^{k} \\
\boldsymbol{\Lambda}
\end{array}\right]=\left[\begin{array}{c}
\mathbf{b}_{1}^{k} \\
\mathbf{b}_{2}^{k} \\
-\mathbf{C}_{1} \mathbf{q}_{1}^{k-1}-\mathbf{C}_{2} \mathbf{q}_{2}^{k-1}
\end{array}\right]
$$

\section{Remark 2}

It may be noted that the appearance of Mortar operators $\mathbf{C}_{s}$ may deteriorate the conditioning of the linear system to be solved at each Gauss-Newton iteration. According to our numerical experiments, the conditioning of the coupled system seems to be reasonable: no numerical instabilities have been encountered. Otherwise, it is possible to scale the Mortar matrices (i.e., to multiply $\mathbf{C}_{s}$ by an adequate value) or to perform a static condensation. We will see in section 5 that a static condensation is performed for the parallel computation of R-DIC, which circumvents the problem of conditioning.

3.2.2. Extension to FE-DIC with mechanical regularization. For the term $\Phi_{r e g}(\mathbf{u})$ in Eq. (8), such a strategy to build the coupling does not directly apply since a norm is not a linear application. Indeed, considering that the displacement is continuous across $\Gamma$, we can only make use of the additivity of vector $\overline{\mathbf{K}} \mathbf{q}$ on $\Omega$ to obtain:

$$
\min _{\mathbf{q}} \frac{1}{2}\left(\|\overline{\mathbf{K}} \mathbf{q}\|_{2}^{2}\right)=\min _{\substack{\mathbf{q}_{1}, \mathbf{q}_{2} \\ \mathbf{q}_{1}=\mathbf{q}_{2} \text { on } \Gamma}} \frac{1}{2}\left(\left\|\overline{\mathbf{K}}_{1} \mathbf{q}_{1}+\overline{\mathbf{K}}_{2} \mathbf{q}_{2}\right\|_{2}^{2}\right),
$$

with:

$$
\left\|\overline{\mathbf{K}}_{1} \mathbf{q}_{1}+\overline{\mathbf{K}}_{2} \mathbf{q}_{2}\right\|_{2}^{2}=\mathbf{q}_{1}^{T} \overline{\mathbf{K}}_{1}^{T} \overline{\mathbf{K}}_{1} \mathbf{q}_{1}+\mathbf{q}_{2}^{T} \overline{\mathbf{K}}_{2}^{T} \overline{\mathbf{K}}_{2} \mathbf{q}_{2}+2 \mathbf{q}_{1}^{T} \overline{\mathbf{K}}_{1}^{T} \overline{\mathbf{K}}_{2} \mathbf{q}_{2}
$$

The problem of directly using expression (17) is that it requires to merge the contributions of the subdomains, which is not compatible with parallel computer architectures. As a consequence, it is proposed instead to consider the following coupling approximation for the minimization of $\Phi_{r e g}(\mathbf{u})$ :

$$
\min _{\mathbf{q}_{1}, \mathbf{q}_{2}} \frac{1}{2}\left(\left\|\overline{\mathbf{K}}_{1} \mathbf{q}_{1}+\overline{\mathbf{K}}_{2} \mathbf{q}_{2}\right\|_{2}^{2}\right) \approx \min _{\substack{\mathbf{q}_{1}, \mathbf{q}_{2}, \overline{\mathbf{\Lambda}} \\ \mathbf{q}_{1}=\mathbf{q}_{2} \text { on } \Gamma}} \frac{1}{2}\left(\left\|\overline{\mathbf{K}}_{1} \mathbf{q}_{1}+\overline{\mathbf{C}}_{1}^{T} \overline{\boldsymbol{\Lambda}}\right\|_{2}^{2}+\left\|\overline{\mathbf{K}}_{2} \mathbf{q}_{2}+\overline{\mathbf{C}}_{2}^{T} \overline{\boldsymbol{\Lambda}}\right\|_{2}^{2}\right) .
$$

In this equation, a new unknown $\bar{\lambda}$ (with corresponding d.o.f vector $\bar{\Lambda}$ ) is introduced to control the mechanical equilibrium at the subdomains interface $\Gamma_{r} . \bar{\lambda}$ is searched for in space $\overline{\mathcal{L}}$ that is spanned by the trace on $\Gamma_{r}$ of the shape functions of $\mathcal{L}$ (see, again, Fig. 2). $\overline{\mathbf{C}}_{s}$ are the associated coupling operators. It has to be noted that the hypothetical case of an exact satisfaction of the mechanical equilibrium between subdomains is recovered with the proposed coupling. In this case, the coupling cost function (18) reaches zero at interface $\Gamma_{r}$ and thus, $\overline{\boldsymbol{\Lambda}}$ exactly corresponds to the interface reaction forces of the two subdomains. However, it has also to be underlined that the proposed coupling is suitable for the more general situation of a displacement $\mathbf{u}$ that does not exactly satisfy the interface equilibrium constraint. Indeed, we emphasize that the static constraint is not enforced exactly as it is done for the displacement (see third term in Eq. (12)), but through a minimization using the $L^{2}$ norm. This allows to represent a little discrepancy between the two interface reaction forces of the two subdomains. This is of crucial importance here because there is no reason that the mechanical residual (18) reaches zero at $\Gamma_{r}$ when combined with the correlation residual (12). 
By performing a weighted sum of the two residuals (12) and (18), we are able to write the version of Eqs. (15) that involves a mechanical regularization of the optical flow. The corresponding linear system reads:

$$
\left[\begin{array}{cccc}
\mathbf{M}_{1}+\eta \overline{\mathbf{K}}_{1}^{T} \overline{\mathbf{K}}_{1} & 0 & \mathbf{C}_{1}^{T} & \eta \overline{\mathbf{K}}_{1}^{T} \overline{\mathbf{C}}_{1}^{T} \\
0 & \mathbf{M}_{2}+\eta \overline{\mathbf{K}}_{2}^{T} \overline{\mathbf{K}}_{2} & \mathbf{C}_{2}^{T} & \eta \overline{\mathbf{K}}_{2}^{T} \overline{\mathbf{C}}_{2}^{T} \\
\mathbf{C}_{1} & \mathbf{C}_{2} & 0 & 0 \\
\eta \overline{\mathbf{C}}_{1} \overline{\mathbf{K}}_{1} & \eta \overline{\mathbf{C}}_{2} \overline{\mathbf{K}}_{2} & 0 & \eta\left(\overline{\mathbf{C}}_{1} \overline{\mathbf{C}}_{1}^{T}+\overline{\mathbf{C}}_{2} \overline{\mathbf{C}}_{2}^{T}\right)
\end{array}\right]\left[\begin{array}{c}
\delta \mathbf{q}_{1}^{k} \\
\delta \mathbf{q}_{2}^{k} \\
\boldsymbol{\Lambda} \\
\overline{\boldsymbol{\Lambda}}
\end{array}\right]=\left[\begin{array}{c}
\mathbf{b}_{1}^{k}-\eta \overline{\mathbf{K}}_{1}^{T} \overline{\mathbf{K}}_{1} \mathbf{q}_{1}^{k-1} \\
\mathbf{b}_{2}^{k}-\eta \overline{\mathbf{K}}_{2}^{T} \overline{\mathbf{K}}_{2} \mathbf{q}_{2}^{k-1} \\
-\mathbf{C}_{1} \mathbf{q}_{1}^{k-1}-\mathbf{C}_{2} \mathbf{q}_{2}^{k-1} \\
-\eta \overline{\mathbf{C}}_{1} \overline{\mathbf{K}}_{1} \mathbf{q}_{1}^{k-1}-\eta \overline{\mathbf{C}}_{2} \overline{\mathbf{K}}_{2} \mathbf{q}_{2}^{k-1}
\end{array}\right]
$$

In practice, the coupling Gauss-Newton algorithm is initialized by the solution of classical FE-DIC problems without mechanical regularization on each subdomain independently. Note that these problems may themselves be initialized by multigrid and coarse gaining techniques $[31,33]$. This provides a good initial estimate since the remaining work performed by the coupling algorithm only consists in imposing the continuity of the displacement across $\Gamma$ and ensuring the mechanical equilibrium of the mechanical models used for regularization.

\section{Remark 3}

Even if it can be interpreted as the interface reaction force, let us notice that the new unknown $\bar{\lambda}$ does not constitute a Lagrange multiplier from a numerical point of view. As a consequence, the situation differs from what we more usually have in computational mechanics, which explains the atypical structure of system (19) (the products of Mortar coupling operators and stiffness matrices appear (see cross terms) and the diagonal term associated to the second interface unknown is not zero).

\section{Remark 4}

It may be noted that there is no evidence that the constructed coupling formulation is consistent with the initial R-DIC problem. This is inherent to the fact that the mechanical equilibrium is controlled through a minimization. Except in the hypothetical case of an exact satisfaction of the interface equilibrium constraint, the equality in Eq. (18) may not be reached. Nevertheless, we were not able to observe any representative difference between the coupling solution (coming from system (19)) and the initial solution (obtained in Eq. (11)) in our numerical experiments (see section 5.3). Whatever the level of regularization is, the method yields the solution of the standard one domain R-DIC problem, which accounts for the reliability of the proposed coupling strategy to be used in RDIC analysis.

\section{APPLICATION TO MULTIRESOLUTION MEASUREMENTS}

As a first application of the proposed method, we address the coupling of multiresolution measurements. The development of a strategy enabling to properly transmit the information between two scales is of crucial interest in any multiscale approach (see, e.g., [20-22] for the context of numerical simulation). In this section, we consider the nearfield/farfield multiscale DIC method introduced in [6]. This method has proved to be efficient for the identification of elastic properties: the farfield measurement is used to provide representative boundary conditions, while the nearfield measurement produces high spatial resolution kinematic fields in a region where the model parameters are sensitive. Nevertheless, the merging of the two scales measurements does not appear trivial in such a situation. In addition to the scaling, a rigid body translation between the farfield and nearfield measurements needs to be controlled. A solution is proposed in the following to answer this issue and the analysis of real multiscale images is performed. For futher details regarding the nearfield/farfield DIC strategy, the interested reader is advised to consult reference [6].

\subsection{Coupling of nearfield/farfield mesurements}

We are now interested in coupling two R-DIC measurements coming from two sets of images that were shot at different magnifications. The experimental setup considered to perform nearfield/farfield DIC is illustrated in Figure 3. The two digital cameras are facing the specimen. Since the optical axis of both cameras need to be perpendicular to the pattern, a translation stage is used to retract the nearfield camera in order to capture the farfield image.

draft

Prepared using nmeauth.cls

Int. J. Numer. Meth. Engng (2016) DOI: $10.1002 / \mathrm{nme}$ 

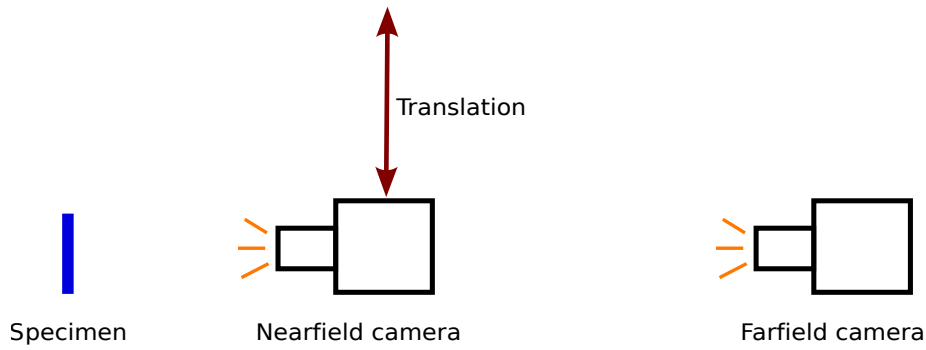

Figure 3. Illustration of the experimental set up to perform nearfield/farfield DIC.

Due to the nearfield camera slide, the nearfield measurement undergoes an arbitrary rigid body translation from the farfield measurement. The interface displacement continuity constraint (see Eq. (13)) needs then to be modified to take into account a rigid body translation between the two scales solutions. Using subscript $n$ (respectively subscript $f$ ) to refer to the nearfield (resp. farfield) measurement and denoting by $\alpha \in \mathbb{R}^{2}$ the in plane rigid body translation between the two scales, Eq. (13) thus becomes:

$$
\int_{\Gamma} \boldsymbol{\lambda} \cdot\left(\mathbf{u}_{n}-\mathbf{u}_{f}+\boldsymbol{\alpha}\right) \mathrm{d} \Gamma=\boldsymbol{\Lambda}^{T}\left(\mathbf{C}_{n} \mathbf{q}_{n}+\mathbf{C}_{f} \mathbf{q}_{f}+\mathbf{R} \boldsymbol{\alpha}\right)
$$

We introduce operator $\mathbf{R}$ for the implementation. Operator $\mathbf{R}$ formally reads as a $\left(n^{\lambda} \times 2\right)$ matrix $\left(n^{\lambda}\right.$ being the number of interface $\Gamma$ d.o.f) with one in the first column if a d.o.f corresponds to a horizontal displacement, one in the second column if a d.o.f corresponds to a vertical displacement, and zero otherwise. By making use of Eq. (20) instead of Eq. (13) to formulate the coupling, we obtain the following linear system to be solved at each Gauss-Newton iteration $k$ :

$$
\left[\begin{array}{cccc}
\mathbf{M}_{n} & 0 & \mathbf{C}_{n}^{T} & 0 \\
0 & \mathbf{M}_{f} & \mathbf{C}_{f}^{T} & 0 \\
\mathbf{C}_{n} & \mathbf{C}_{f} & 0 & \mathbf{R} \\
0 & 0 & \mathbf{R}^{T} & 0
\end{array}\right]\left[\begin{array}{c}
\delta \mathbf{q}_{n}^{k} \\
\delta \mathbf{q}_{f}^{k} \\
\boldsymbol{\Lambda} \\
\delta \boldsymbol{\alpha}^{k}
\end{array}\right]=\left[\begin{array}{c}
\mathbf{b}_{n}^{k} \\
\mathbf{b}_{f}^{k} \\
-\mathbf{C}_{n} \mathbf{q}_{n}^{k-1}-\mathbf{C}_{f} \mathbf{q}_{f}^{k-1}-\mathbf{R} \boldsymbol{\alpha}^{k-1} \\
0
\end{array}\right]
$$

System (21) characterizes the nearfield/farfield coupling algorithm without mechanical regularization. Note that it enables not only to compute a continuous displacement solution through the nearfield and farfield images but also to evaluate the rigid body translation between the two measurements. Indeed, starting with an initial estimate $\boldsymbol{\alpha}^{0}$ of the rigid body translation, a correction $\delta \boldsymbol{\alpha}^{k}$ of the rigid mode is computed at each correlation iteration $k$. For the initialization of the nearfield/farfield coupling algorithm, we proceed similarly as in previous section. The two FE-DIC problems associated to the two scales are first solved independently, which gives the initalizations $\mathbf{q}_{n}^{0}$ and $\mathbf{q}_{f}^{0}$. From these two independent solutions, the initial rigid body translation is then estimated by minimizing the kinematic constraint in a least square sense:

$$
\mathbf{R}^{T} \mathbf{R} \boldsymbol{\alpha}^{0}=-\mathbf{R}^{T}\left(\mathbf{C}_{n} \mathbf{q}_{n}^{0}+\mathbf{C}_{f} \mathbf{q}_{f}^{0}\right)
$$

Until now, no mechanical regularization of the optical flow has been performed. The extension to multiscale DIC with mechanical regularization is straightforward with the developments of section 3.2. The new system to be 
solved at each Gauss-Newton iteration $k$ reads:

$$
\begin{aligned}
& {\left[\begin{array}{ccccc}
\mathbf{M}_{n}+\eta \overline{\mathbf{K}}_{n}^{T} \overline{\mathbf{K}}_{n} & 0 & \mathbf{C}_{n}^{T} & \eta \overline{\mathbf{K}}_{n}^{T} \overline{\mathbf{C}}_{n}^{T} & 0 \\
0 & \mathbf{M}_{f}+\eta \overline{\mathbf{K}}_{f}^{T} \overline{\mathbf{K}}_{f} & \mathbf{C}_{f}^{T} & \eta \overline{\mathbf{K}}_{f}^{T} \overline{\mathbf{C}}_{f}^{T} & 0 \\
\mathbf{C}_{n} & \mathbf{C}_{f} & 0 & 0 & \mathbf{R} \\
\eta \overline{\mathbf{C}}_{n} \overline{\mathbf{K}}_{n} & \eta \overline{\mathbf{C}}_{f} \overline{\mathbf{K}}_{f} & 0 & \eta\left(\overline{\mathbf{C}}_{n} \overline{\mathbf{C}}_{n}^{T}+\overline{\mathbf{C}}_{f} \overline{\mathbf{C}}_{f}^{T}\right) & 0 \\
0 & 0 & \mathbf{R}^{T} & 0 & 0
\end{array}\right]\left[\begin{array}{c}
\delta \mathbf{q}_{n}^{k} \\
\delta \mathbf{q}_{f}^{k} \\
\boldsymbol{\Lambda} \\
\overline{\boldsymbol{\Lambda}} \\
\delta \boldsymbol{\alpha}^{k}
\end{array}\right]=} \\
& {\left[\begin{array}{c}
\mathbf{b}_{n}^{k}-\eta \overline{\mathbf{K}}_{n}^{T} \overline{\mathbf{K}}_{n} \mathbf{q}_{n}^{k-1} \\
\mathbf{b}_{f}^{k}-\eta \overline{\mathbf{K}}_{f}^{T} \overline{\mathbf{K}}_{f} \mathbf{q}_{f}^{k-1} \\
-\mathbf{C}_{n} \mathbf{q}_{n}^{k-1}-\mathbf{C}_{f} \mathbf{q}_{f}^{k-1}-\mathbf{R} \boldsymbol{\alpha}^{k-1} \\
-\eta \overline{\mathbf{C}}_{n} \overline{\mathbf{K}}_{n} \mathbf{q}_{n}^{k-1}-\eta \overline{\mathbf{C}}_{f} \overline{\mathbf{K}}_{f} \mathbf{q}_{f}^{k-1} \\
0
\end{array}\right]}
\end{aligned}
$$

\section{Remark 5}

With the experimental set up of Figure 3, one may notice that the large scale data corresponding to the local subregion are available and could thus be used as additional information to solve the multiscale correlation problem. For simplicity, this feature has not been considered in this preliminary work but it may deserve attention in future investigations.

\subsection{Analysis of real multiresolution images}

The proposed multiscale coupling methodology is now applied to the analysis of a tensile test performed on an open hole glass/epoxy composite specimen first analysed in [6]. A black and white speckle is sprayed on top of the surface (the speckle is intentionally made finer in the nearfield region) and two cameras are used to capture images $(1392 \times 1040$ pixels). The first camera takes pictures of the whole gauge region, while the second focus over a smaller area located around the hole. The ratio between the two optical resolutions is around 5. An unstructured finite element mesh made of linear quadrangles is adjusted on the images (in practice, it is first adjusted on the farfield image and then automatically adjusted to the reference nearfield image using a image-based calibration performed beforehand [6]). For the computation, only the elements that are not concerned by the nearfield measurement are taken into account in the farfield measurement. Further details regarding the experimental set up can be found in [6].

The corresponding measured FE-DIC displacement fields along the transverse direction are presented in Figure 4. An amplification factor of 30 is applied for the deformed configurations. More precisely, Figure 4(a) first shows the plot of intializations $\mathbf{q}_{n}^{0}$ and $\mathbf{q}_{f}^{0}$. We clearly see that the nearfield measurement undergoes a rigid body translation from the farfield measurement. By removing the initial rigid body translation $\boldsymbol{\alpha}^{0}$ (see Eq. (22)) from the nearfield measurement, the solution of Figure 4(b) is obtained. It can be observed that the nearfield solution is replaced within the farfield solution but that the displacement continuity between the two scales measurements is not verified, which is not compatible with FE-DIC. In addition, it can be noticed that the displacement is more regular in the nearfield region. This is expected since a higher image resolution is considered in this region. From this initialization, the multiscale coupling algorithm (21) is applied which leads to the solution shown in Figure 4(c). The displacement field becomes perfectly continuous across the multiscale coupling interface. Nevertheless, the solution seems to be subjected to spurious oscillations around the interface. We finally add a mechanical regularization of the optical flow in Figure 4(d) $\left(\eta=10^{-5}\right)$. In order to do so, algorithm (23) is performed from the initialization of Figure 4(b). We observe that it results in a more regular solution. In opposition to the displacement continuity which is prescribed exactly, the mechanical regularization is prescribed in a week manner and so is the equilibrium between scales. However, the transition of the measured displacement field from the two scales appears very smooth: the spurious oscillations around the interface have disappeared. This accounts for the effectiveness of proposed method to couple multiresolution measurements. 

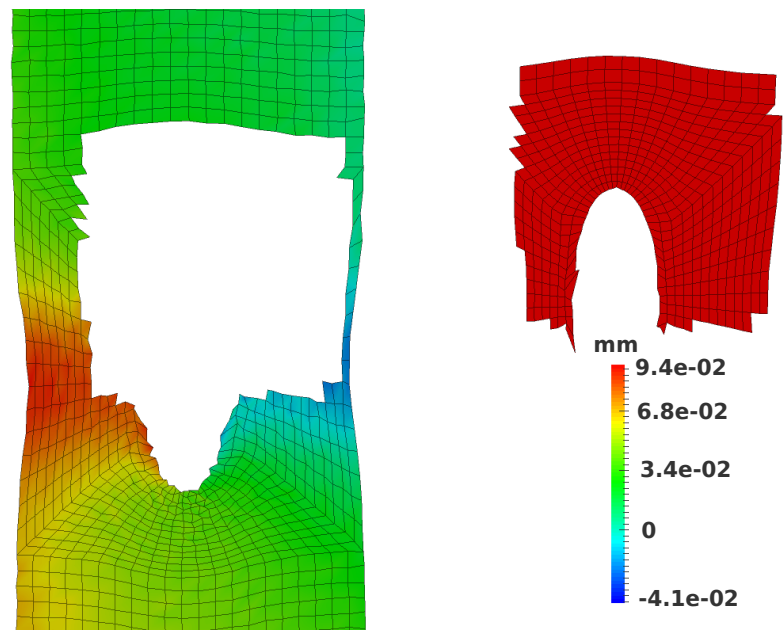

(a) Solution (without mechanical regularization) computed independently on each scale.

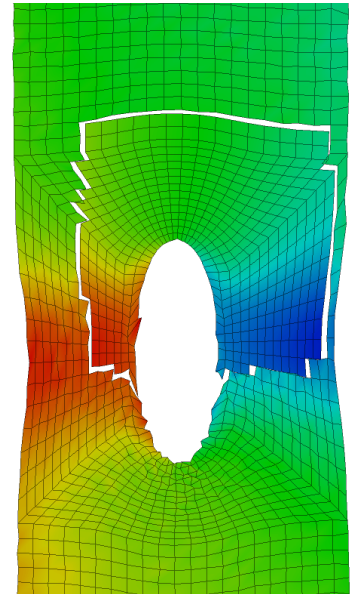

(b) Initialization of the coupling algorithm: removal of the rigid body translation (see Eq. (22)).

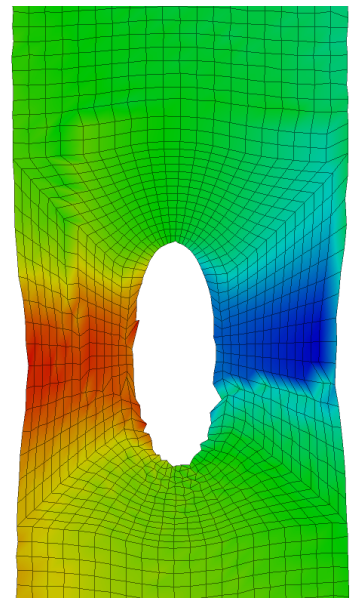

(c) Continuous solution wihtout mechanical regularization obtained with the coupling algorithm (21).

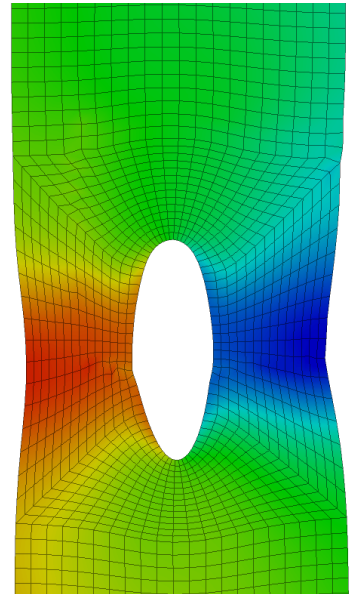

(d) Continuous solution wiht mechanical regularization obtained with the coupling algorithm (23).

Figure 4. Horizontal displacement solution (in mm, amplification factor of 30) measured by nearfield/farfield FE-DIC for the tensile test.

\section{APPLICATION TO HIGH-PERFORMANCE COMPUTING}

As mentionned at the end of section 2.1, the drawback of FE-DIC over subset-DIC is the computational cost when high resolution measurements on large images are required. The computational burden associated to FE-DIC in such situations is mostly due to the inversion of the finite element systems (11) and to image interpolations (required to compute the right hand side $\mathbf{b}^{k}$, see Eq. (7)). As demonstrated in [23], a dual domain decomposition method for FE-DIC can be implemented to combine the advantages of FE-DIC (direct bridge with simulation, continuity, mechanical regularization...) and subset-DIC (parallelization ability). In former contribution [23], only the case of FE-DIC without mechanical regularization has been investigated. By making use of the newly developed coupling, we now extend the strategy for the parallel computation of R-DIC. We proceed as follows: first, the interface problem resulting from the proposed coupling is written, then; an iterative algorithm allowing for highperformance computing is built, finally; the method is used to analyze high resolution images of a real experiment. 


\subsection{Construction of the interface problem}

Since the initial system to be solved involves the FE stiffness matrix K (see Eq. (11)), our development is based on works conducted on non-overlapping domain decomposition methods in structural mechanics (see, e.g., [34-40]). The starting point is to write the interface problem associated to the coupling. In order to do so, system (19) is first split into the following coupled equations:

$$
\begin{aligned}
& \delta \mathbf{q}_{s}^{k}=\left(\mathbf{M}_{s}+\eta \overline{\mathbf{K}}_{s}^{T} \overline{\mathbf{K}}_{s}\right)^{-1}\left(\mathbf{b}_{s}^{k}-\eta \overline{\mathbf{K}}_{s}^{T} \overline{\mathbf{K}}_{s} \mathbf{q}_{s}^{k-1}-\mathbf{C}_{s}^{T} \boldsymbol{\Lambda}-\eta \overline{\mathbf{K}}_{s}^{T} \overline{\mathbf{C}}_{s}^{T} \overline{\boldsymbol{\Lambda}}\right) \quad \forall s \\
& \sum_{s} \mathbf{C}_{s}\left(\delta \mathbf{q}_{s}^{k}+\mathbf{q}_{s}^{k-1}\right)=0 \\
& \sum_{s} \eta \overline{\mathbf{C}}_{s}\left(\overline{\mathbf{K}}_{s}\left(\delta \mathbf{q}_{s}^{k}+\mathbf{q}_{s}^{k-1}\right)+\overline{\mathbf{C}}_{s}^{T} \overline{\boldsymbol{\Lambda}}\right)=0
\end{aligned}
$$

assuming now an arbitrary number of subdomains. By substituting Eq. (24a) into Eq. (24b), the coupled problem can then be condensed onto the interface d.o.f., which gives:

$$
\mathbb{S}_{t o t}^{d} \boldsymbol{\Lambda}_{t o t}=\mathbf{t}_{t o t}^{k} \text {. }
$$

The so-called dual Schur complement operator $\mathbb{S}_{t o t}^{d}$, the total Lagrange multiplier d.o.f vector $\boldsymbol{\Lambda}_{t o t}$, and the condensed right hand side $\mathbf{t}_{t o t}^{k}$ read as follows:

$$
\mathbb{S}_{t o t}^{d}=\left[\begin{array}{cc}
\mathbf{S}^{\lambda \lambda} & \mathbf{S}^{\lambda \bar{\lambda}} \\
\left(\mathbf{S}^{\lambda \bar{\lambda}}\right)^{T} & \mathbf{S}^{\overline{\lambda \lambda}}
\end{array}\right] ; \boldsymbol{\Lambda}_{t o t}=\left[\begin{array}{c}
\boldsymbol{\Lambda} \\
\overline{\boldsymbol{\Lambda}}
\end{array}\right] \quad \text { and } \quad \mathbf{t}_{t o t}^{k}=\left[\begin{array}{c}
\mathbf{t}^{k} \\
\overline{\mathbf{t}}^{k}
\end{array}\right],
$$

where, denoting by $\mathbf{I}_{s}$ the local identity matrix (whose size is the number of local displacement d.o.f.):

$$
\begin{aligned}
& \mathbf{S}^{\lambda \lambda}=\sum_{s} \mathbf{C}_{s}\left(\mathbf{M}_{s}+\eta \overline{\mathbf{K}}_{s}^{T} \overline{\mathbf{K}}_{s}\right)^{-1} \mathbf{C}_{s}^{T} ; \\
& \mathbf{S}^{\lambda \bar{\lambda}}=\sum_{s} \mathbf{C}_{s}\left(\mathbf{M}_{s}+\eta \overline{\mathbf{K}}_{s}^{T} \overline{\mathbf{K}}_{s}\right)^{-1} \eta \overline{\mathbf{K}}_{s}^{T} \overline{\mathbf{C}}_{s}^{T} ; \\
& \mathbf{S}^{\overline{\lambda \lambda}}=\sum_{s} \eta \overline{\mathbf{C}}_{s}\left(\overline{\mathbf{K}}_{s}\left(\mathbf{M}_{s}+\eta \overline{\mathbf{K}}_{s}^{T} \overline{\mathbf{K}}_{s}\right)^{-1} \eta \overline{\mathbf{K}}_{s}^{T}-\mathbf{I}_{s}\right) \overline{\mathbf{C}}_{s}^{T} ; \\
& \mathbf{t}^{k}=\sum_{s} \mathbf{C}_{s}\left(\mathbf{q}_{s}^{k-1}+\left(\mathbf{M}_{s}+\eta \overline{\mathbf{K}}_{s}^{T} \overline{\mathbf{K}}_{s}\right)^{-1}\left(\mathbf{b}_{s}^{k}-\eta \overline{\mathbf{K}}_{s}^{T} \overline{\mathbf{K}}_{s} \mathbf{q}_{s}^{k-1}\right)\right) ; \\
& \overline{\mathbf{t}}^{k}=\sum_{s} \eta \overline{\mathbf{C}}_{s} \overline{\mathbf{K}}_{s}\left(\mathbf{q}_{s}^{k-1}+\left(\mathbf{M}_{s}+\eta \overline{\mathbf{K}}_{s}^{T} \overline{\mathbf{K}}_{s}\right)^{-1}\left(\mathbf{b}_{s}^{k}-\eta \overline{\mathbf{K}}_{s}^{T} \overline{\mathbf{K}}_{s} \mathbf{q}_{s}^{k-1}\right)\right) .
\end{aligned}
$$

\section{Remark 6}

When more than two subdomains are considered, attention must be paid for the coupling to the interface d.o.f. belonging to more than two subdomains (multiplicity greater than 2). In this situation, we choose to implement a non-redundant connectivity (see, e.g., [34] for completeness).

\section{Remark 7}

Let us finally notice that the dual Schur complement operator $\mathbb{S}_{t o t}^{d}$ is symmetric but non positive definite although the initial correlation operator is. Once again, one must keep in mind that $\bar{\Lambda}$ does not constitute a Lagrange multiplier, which is responsible for these atypical developments.

\subsection{Parallel resolution}

5.2.1. Iterative solution algorithm. With above developments, we now need (at each Gauss-Newton iteration $k$ ) to solve interface problem (25) and to apply Eq. (24a) to obtain the Gauss-Newton update. Following the domain decomposition framework, interface problem (25) is solved using a Krylov iterative solver (see, e.g., [41-44]) in 
order to fit parallelization criteria. Starting with initialization $\boldsymbol{\Lambda}_{\text {tot }}^{(0)}$ and associated residual $\mathbf{r}_{\text {tot }}^{(0)}=\mathbb{S}_{\text {tot }}^{d} \boldsymbol{\Lambda}_{\text {tot }}^{(0)}-\mathbf{t}_{\text {tot }}^{k}$, Krylov solvers are based on the iterative construction of so-called "Krylov subspace" $\mathcal{K}_{m}\left(\mathbb{S}_{\text {tot }}^{d}, \mathbf{r}_{\text {tot }}^{(0)}\right)$ defined by:

$$
\mathcal{K}_{m}\left(\mathbb{S}_{\text {tot }}^{d}, \mathbf{r}_{\text {tot }}^{(0)}\right)=\operatorname{span}\left[\mathbf{r}_{\text {tot }}^{(0)}, \ldots,\left(\mathbb{S}_{\text {tot }}^{d}\right)^{m-1} \mathbf{r}_{\text {tot }}^{(0)}\right]
$$

Thus, each Krylov iteration $j$ requires to perform a matrix-vector product involving operator $\mathbb{S}_{t o t}^{d}$. Let us denote by $\boldsymbol{v}_{t o t}$ any vector of the size of the interface problem such that:

$$
\boldsymbol{v}_{t o t}=\left[\begin{array}{c}
\boldsymbol{v} \\
\overline{\boldsymbol{v}}
\end{array}\right] \text {. }
$$

The matrix-vector product $\mathbb{S}_{\text {tot }}^{d} \boldsymbol{v}_{\text {tot }}$ corresponds to the following computation:

$$
\mathbb{S}_{t o t}^{d} \boldsymbol{v}_{t o t}=\left[\begin{array}{c}
\sum_{s} \mathbf{C}_{s}\left(\mathbf{y}_{s}+\overline{\mathbf{y}}_{s}\right) \\
\sum_{s} \eta \overline{\mathbf{C}}_{s} \overline{\mathbf{K}}_{s}\left(\mathbf{y}_{s}+\overline{\mathbf{y}}_{s}\right)-\sum_{s} \eta \overline{\mathbf{C}}_{s} \overline{\mathbf{C}}_{s}^{T} \overline{\boldsymbol{v}}
\end{array}\right],
$$

where $\mathbf{y}_{s}$ and $\overline{\mathbf{y}}_{s}$ are the solutions of the set of local (i.e., independent on each subdomain) systems :

$$
\left\{\begin{array}{l}
\left(\mathbf{M}_{s}+\eta \overline{\mathbf{K}}_{s}^{T} \overline{\mathbf{K}}_{s}\right) \mathbf{y}_{s}=\mathbf{C}_{s}^{T} \boldsymbol{v} \\
\left(\mathbf{M}_{s}+\eta \overline{\mathbf{K}}_{s}^{T} \overline{\mathbf{K}}_{s}\right) \overline{\mathbf{y}}_{s}=\eta \overline{\mathbf{K}}_{s}^{T} \overline{\mathbf{C}}_{s}^{T} \overline{\boldsymbol{v}}
\end{array} .\right.
$$

These systems correspond to local (mechanically regularized) FE-DIC problems with different right hand sides. Since they are independent from one subdomain to the other, these system resolutions (in addition to be computationally affordable because restricted to subdomains) can be performed in parallel. This is the key point of domain decomposition methods: parallel processing can be used when computing matrix-vector products of form $\mathbb{S}_{t o t}^{d} \boldsymbol{v}_{t o t}$. This enables to limit the computation cost and the storage requirement to solve, at each Gauss-Newton iteration $k$, linear system (11). In practice, a direct solver is used for the local resolutions (31). Since the right hand side $\mathbf{t}_{t o t}^{k}$ is the sum of local contributions, it can also be computed in parallel (to evaluate the residuals). Finally, note that operators $\mathbf{M}_{s}+\eta \overline{\mathbf{K}}_{s}^{T} \overline{\mathbf{K}}_{s}$ remain unchanged during correlation iterations. Thus, they are factorized once at the beginning.

Since matrix $\mathbb{S}_{t o t}^{d}$ is symmetric but non positive definite, a preconditioned GMRES algorithm [43] is used as the Krylov solver. Algorithm 1 describes the implemented procedure. Operator $\mathbb{P}_{i}$ is introduced as a preconditioner. Preconditioning will be discussed in next section. At each iteration $j$, a full orthonormalization of vector $\boldsymbol{w}_{t o t}^{(j)}$ with respect to $\mathcal{K}_{j}$ is performed using a modified Gram-Schmidt algorithm. $\mathbf{e}_{1}$ is a vector of size $j+1$. Only its first component does not vanish: it is set to 1 . We note $\varepsilon$ the stopping tolerance of the algorithm. It is proposed to take the norm of the residual of the initial problem $\mathbb{S}_{t o t}^{d} \boldsymbol{\Lambda}_{t o t}=\mathbf{t}_{t o t}^{k}$ for the stopping criterion. When used with preconditioning, this requires to compute, at each iteration $j$, a least square problem to evaluate the $j^{t h}$ approximation $\boldsymbol{\Lambda}_{\text {tot }}^{(j)}$ (see Step 11 of Alg. 1). It has to be noted that a smart implementation of GMRES enables to directly access the norm of the residual $\mathbf{r}_{t o t}^{(j)}$ without computing $\boldsymbol{\Lambda}_{t o t}^{(j)}$. Thus, Steps 9 to 11 of Alg. 1 can be performed only once (at the end of the algorithm). The problem of such a strategy when used with preconditioning is that the residual depends on the preconditioner, which makes difficult the comparison between different acceleration techniques. For this reason, we preferred to use the residual of the initial problem independently of the preconditioner employed.

\section{Remark 8}

Since operator $\mathbb{S}_{t o t}^{d}$ is symmetric, it may be noted that a MINRES or a SYMMLQ algorithm could also be suitable for the iterative resolution (see, e.g., [42] for a review). Nevertheless, we were not able to get satisfactory results with these algorithms to solve system (25) that is why we preferred to implement the more usual GMRES algorithm.

We conclude this part by adding some remarks regarding the global implementation of the method. Each of the points mentioned below have been discussed in former contribution [23] so we advise the interested reader to consult this reference for more completeness. First and foremost, it has to be noted that not only the system 


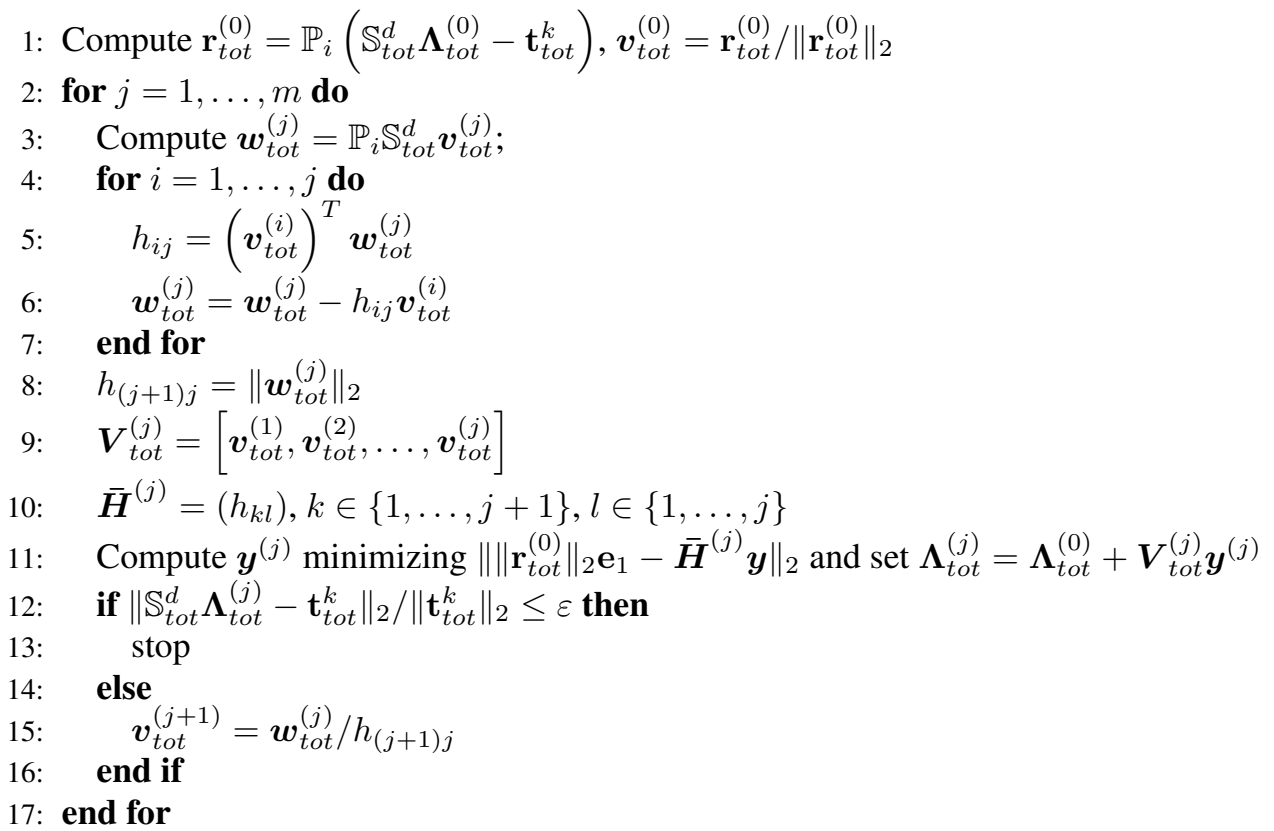

Algorithm 1: Preconditioned GMRES algorithm implemented to solve interface problem (25).

inversions but also the image interpolations can be performed in parallel since both the mesh and the images are subdivided. Then, it has to be recalled that the constructed mesh and images subdivisions are used to perform the initialization of the coupling Gauss-Newton on each subdomain independently. This stage is highly parallelisable because no communications are required between subdomains. Finally, we proceed as follows for the initialization of the Krylov iterative solver: at first correlation iteration $(k=0), \Lambda_{t o t}^{(0)}$ is set to zero; then, for the next correlation iterations $(k>0), \Lambda_{t o t}^{(0)}$ is set to the last value of the previous GMRES resolution. This is a simple but efficient way to reduce the number of Krylov iterations. An overview of the proposed domain decomposition FE-DIC (DD FE-DIC) algorithm is given in Figure 5 for illustration.

5.2.2. Convergence acceleration. We now address preconditioning in order to speed up the GMRES resolution (Alg. 1). The objective of this part is to find an interface operator $\mathbb{P}_{i}$ that is cheap to construct and that gives a good approximation of the inverse of $\mathbb{S}_{d}^{t o t}$. Once again, we propose to follow the approach introduced in non-overlapping domain decomposition methods in structural mechanics (see [34] for a review) to build a relevant preconditioner. The strategy is based on the resolution of local problems.

From here on, we use the following notation for the local correlation operator:

$$
\mathbf{M} \eta \overline{\mathbf{K}}_{s}=\mathbf{M}_{s}+\eta \overline{\mathbf{K}}_{s}^{T} \overline{\mathbf{K}}_{s}
$$

We also introduce new operators $\mathbf{c}_{s}, \overline{\mathbf{c}}_{s}, \mathbf{A}_{s}$ and $\overline{\mathbf{A}}_{s}$ such that:

$$
\mathbf{C}_{s}=\mathbf{A}_{s} \mathbf{c}_{s} \text { and } \overline{\mathbf{C}}_{s}=\overline{\mathbf{A}}_{s} \overline{\mathbf{c}}_{s} .
$$

$\mathbf{c}_{s}$ (respectively $\overline{\mathbf{c}}_{s}$ ) is the local trace operator which projects the $n_{s}^{u}$ local displacements of $\Omega_{s}$ onto the $n_{s}^{\lambda}$ (resp. $n_{s}^{\bar{\lambda}}$ ) local interface displacements of $\Gamma_{s}$ (resp. $\Gamma_{r_{s}}$ ). $\mathbf{A}_{s}$ and $\overline{\mathbf{A}}_{s}$ are the associated assembly operators that exchange data lying on one subdomain interface with its neighboring subdomains. Using above notations, operator $\mathbb{S}_{\text {tot }}^{d}$ can be rewritten as follows:

$$
\mathbb{S}_{t o t}^{d}=\sum_{s} \mathbb{A}_{s} \mathbb{S}_{s}^{d} \mathbb{A}_{s}^{T}
$$

with:

$$
\mathbb{A}_{s}=\left[\begin{array}{cc}
\mathbf{A}_{s} & \mathbf{0} \\
\mathbf{0} & \overline{\mathbf{A}}_{s}
\end{array}\right] ; \mathbb{S}_{s}^{d}=\left[\begin{array}{cc}
\mathbf{S}_{s}^{\lambda \lambda} & \mathbf{S}_{s}^{\lambda \bar{\lambda}} \\
\left(\mathbf{S}_{s}^{\lambda \bar{\lambda}}\right)^{T} & \mathbf{S}_{s}^{\overline{\lambda \lambda}}
\end{array}\right]
$$




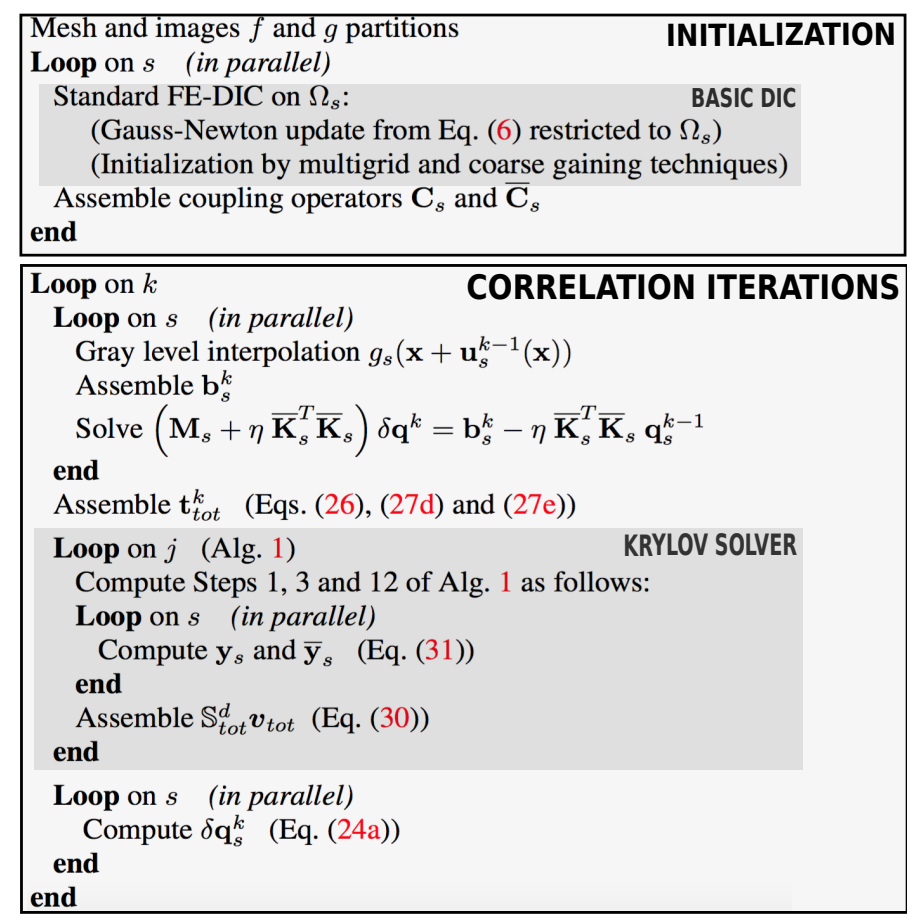

Figure 5. Overview of the proposed DD FE-DIC algorithm.

and where:

$$
\begin{aligned}
& \mathbf{S}_{s}^{\lambda \lambda}=\mathbf{c}_{s}\left(\mathbf{M} \eta \overline{\mathbf{K}}_{s}\right)^{-1} \mathbf{c}_{s}^{T} ; \\
& \mathbf{S}_{s}^{\lambda \bar{\lambda}}=\mathbf{c}_{s}\left(\mathbf{M} \eta \overline{\mathbf{K}}_{s}\right)^{-1} \eta \overline{\mathbf{K}}_{s}^{T} \overline{\mathbf{c}}_{s}^{T} ; \\
& \mathbf{S}_{s}^{\overline{\lambda \lambda}}=\eta \overline{\mathbf{c}}_{s}\left(\overline{\mathbf{K}}_{s}\left(\mathbf{M} \eta \overline{\mathbf{K}}_{s}\right)^{-1} \eta \overline{\mathbf{K}}_{s}^{T}-\mathbf{I}_{s}\right) \overline{\mathbf{c}}_{s}^{T} .
\end{aligned}
$$

Since operator $\mathbb{S}_{t o t}^{d}$ (Eq. (34)) is the sum of local contributions, the classical strategy following domain decomposition in structural mechanics is to define $\mathbb{P}_{i}$ as a sum of the inverse of local contributions:

$$
\mathbb{P}_{i}=\sum_{s} \mathbb{A}_{s}\left(\mathbb{S}_{s}^{d}\right)^{-1} \mathbb{A}_{s}^{T}
$$

The difficulty with the proposed formulation which is not purely based on a Lagrangian (due to $\bar{\lambda}$ ) is that we cannot directly form a local primal local Schur complement equals to $\left(\mathbb{S}_{s}^{d}\right)^{-1}$. As a result, we propose at this step to explicitly compute the local inverse as follows:

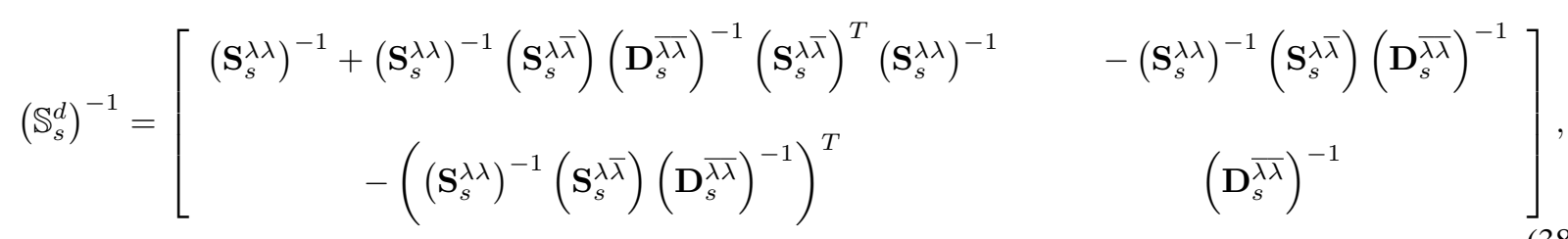

with:

$$
\mathbf{D}_{s}^{\overline{\lambda \lambda}}=\mathbf{S}_{s}^{\overline{\lambda \lambda}}-\left(\mathbf{S}_{s}^{\lambda \bar{\lambda}}\right)^{T}\left(\mathbf{S}_{s}^{\lambda \lambda}\right)^{-1}\left(\mathbf{S}_{s}^{\lambda \bar{\lambda}}\right)
$$

It has to be noted that the introduction of a preconditioner in algorithm 1 only requires matrix-vector products involving $\mathbb{P}_{i}$ (see Steps 1 and 3). With the proposed form of the preconditioner (Eqs. (37)-(39)), this implies to explicitly compute $\mathbf{D}_{s}^{\overline{\lambda \lambda}}$ so that, a factorization of $\mathbf{D}_{s}^{\overline{\lambda \lambda}}$ can be performed and used for local resolutions in parallel 
(similar procedure as for the computation of $\mathbb{S}_{t o t}^{d} \boldsymbol{v}_{t o t}$, see Eqs. (30) and (31)). The interesting point here is to notice that a simple expression of $\left(\mathbf{S}_{s}^{\lambda \lambda}\right)^{-1}$ can be obtained. Denoting by $[\square]_{i}$ any restriction to internal data of a local quantity $\square$ and by $[\square]_{b}$ the restriction to interface data (see Figure 6 for illustration), we can write:

$$
\left(\mathbf{S}_{s}^{\lambda \lambda}\right)^{-1}=\left(\mathbf{c}_{s}\left(\mathbf{M} \eta \overline{\mathbf{K}}_{s}\right)^{-1} \mathbf{c}_{s}^{T}\right)^{-1}=\left[\mathbf{M} \eta \overline{\mathbf{K}}_{s}\right]_{b b}-\left[\mathbf{M} \eta \overline{\mathbf{K}}_{s}\right]_{b i}\left(\left[\mathbf{M} \eta \overline{\mathbf{K}}_{s}\right]_{i i}\right)^{-1}\left[\mathbf{M} \eta \overline{\mathbf{K}}_{s}\right]_{i b} .
$$

This equality is derived from results based on local primal and dual Schur complements [34]. The computational cost for evaluating $\left(\mathbf{S}_{s}^{\lambda \lambda}\right)^{-1}$ is therefore related to the computation of term $\left(\left[\mathbf{M} \eta \overline{\mathbf{K}}_{s}\right]_{i i}\right)^{-1}\left[\mathbf{M} \eta \overline{\mathbf{K}}_{s}\right]_{i b}:$ it requires to factorize $\left[\mathbf{M} \eta \overline{\mathbf{K}}_{s}\right]_{i i}$ and to solve $n_{s}^{\lambda}$ linear systems of size $n_{s}^{u_{i}} \times n_{s}^{u_{i}}\left(n_{s}^{u_{i}}\right.$ being the number of local internal displacements). This is affordable because (i) it has to be done once at the beginning of the Gauss-Newton algorithm, (ii) it is local and thus, (iii) it can be done in parallel between subdomains. In addition, we need to evaluate $\mathbf{S}_{s}^{\overline{\lambda \lambda}}$ and $\mathbf{S}_{s}^{\lambda \bar{\lambda}}$. This requires to compute $\left(\mathbf{M} \eta \overline{\mathbf{K}}_{s}\right)^{-1} \overline{\mathbf{K}}_{\mathbf{s}}{ }^{T} \overline{\mathbf{c}}_{\mathbf{s}}{ }^{T}$ which is affordable as well. The factorization of $\mathbf{M} \eta \overline{\mathbf{K}}_{s}$ (already performed to solve (31)) is used for that and $n_{s}^{\bar{\lambda}}$ linear systems of size $n_{s}^{u} \times n_{s}^{u}$ are solved.

As a result, we end up with a strategy that involves only local resolutions and that is completely parallelisable. A slight additional cost is then necessary to apply the proposed preconditioning but it leads to a drastic reduction of the number of GMRES iterations, as shown in the examples below.

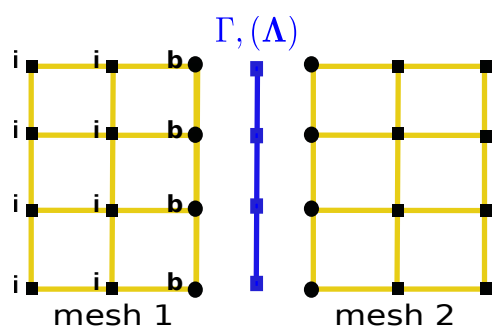

Figure 6. Internal and $\Gamma$ interface numbering for the two subdomains decomposition.

\section{Remark 9}

Besides preconditioning, it could be of interest to reuse Krylov subspaces [44] to accelerate the GMRES resolution. This could be particularly efficient here because the operator is constant and the right hand side changes little.

\subsection{Application to a four point bending test}

5.3.1. Experimental set up and associated domain decomposition model. The developed DD FE-DIC method is now applied to the analysis of high resolution images of a real experiment. A four point bending test is performed on an open hole polymethyl methacrylate specimen (see Figure 7). More precisely, the pattern consists of a parallelepipedic coupon of dimension $200 \times 20 \times 5 \mathrm{~mm}$ with an $8 \mathrm{~mm}$ hole drilled in the center. A black and white speckle pattern is sprayed onto the surface of the specimen and a high resolution CCD camera (29 megapixels) is used to capture images ( $4384 \times 6576$ pixels). The physical size of one pixel is $19.4 \mu \mathrm{m}$. An unstructured finite element mesh (including both linear triangles and quadrangles of 15 pixels width in average) is adjusted on the image (see Figure 8) to perform FE-DIC. An elastic regularization of the optical flow is adopted $\left(\eta=10^{-3}\right)$. Figure 7 specifies the boundary nodes that are not mechanically controlled. The mesh and images are decomposed into 180 subdomains for the DD computation. The resulting average subdomain size is 160 pixels per side. With such a discretization, the displacement field includes 62522 d.o.f. and the interface problem (25) contains 21174 interface d.o.f (distributed as follows: 10604 d.o.f. for $\boldsymbol{\Lambda}$ and 10570 d.o.f for $\bar{\Lambda}$ ). The analysis is finally performed with a stopping tolerance for correlation and Krylov iterations of $10^{-3}$.

5.3.2. Domain decomposition solution. Figure 9 shows the deformed configurations obtained with the considered domain decomposition (amplification factor of 50). The color scale corresponds to the horizontal component of the displacement field. More precisely, the solution associated to the parallel initialization of the coupling GaussNewton algorithm (i.e., where classical FE-DIC problems without mechanical regularization are solved on each subdomain independently) is first plotted (see Figure 9(a)). From this initialization, the solution obtained using the 


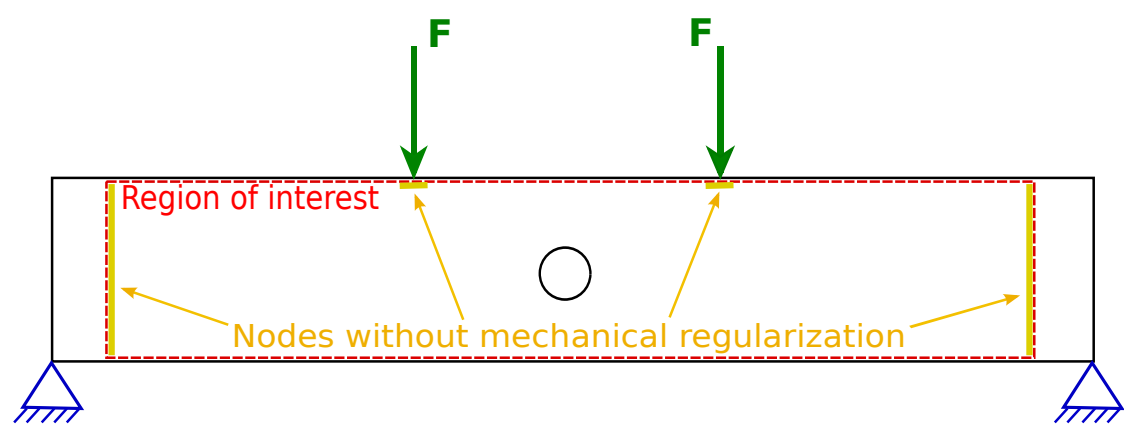

Figure 7. Four point bending test: illustration of the experimental set up.

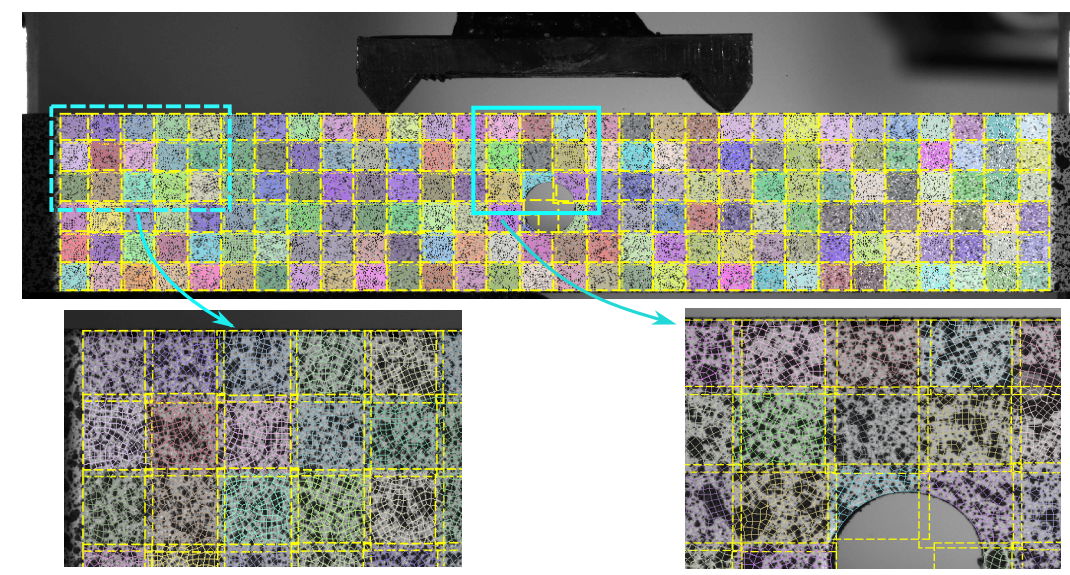

Figure 8. Reference image and domain decomposition (180 subdomains) for the four point bending test. The overlapping subset images $f_{s}$ are in yellow dashed line. The finite elements belonging to a same subdomain are plotted with the same colour. Two zooms are provided below the image.

domain decompostion method of [23] is shown in Figure 9(b) while Figure 9(c) presents the solution computed with the proposed domain decomposition algorithm.

We clearly see the improvement of the solution through the different situations. In the initial solution (Figure 9(a)), the displacement continuity is not verified and sharp oscillations are present in the measured field. Nevertheless, the solution constitutes a good initialization of the proposed domain decompostion algorithm: the global displacement field at the interior of the subdomains does not seem too far from the intended solution. With the domain decomposition algorithm of [23], the displacement becomes continuous across the subdomains but the field measurement still suffers from some instabilities which leads to a non-smooth solution (see Figure 9(b)). When performing the proposed domain decomposition algorithm, not only the displacement becomes continuous across the subdomains but also the mechanical equilibrium of the structure is ensured which removes the oscillations of the measured field (see Figure 9(c)). As a result, the proposed DD FE-DIC strategy enables to compute in parallel an accurate mechanically sound solution from high resolution images.

Following remark 4, we also numerically assess the consistency of the proposed coupling with respect to the initial FE-DIC problem. In order to do so, we compute an error map between the DD solution with mechanical regularization (see Figure 9(c)) and the corresponding mono-domain solution (coming from Eq. (11)). Since in the DD solution, more than one displacement exists at interface points, the DD solution at the interface is chosen to be the average of the solution of all the neighboring sub-domains. The result is a vector field. The $x$-component of the relative error map (normalized by the maximum $x$-displacement) is plotted in figure 10 . The color scale is rescaled between 0 and $10^{-3}$. We recall that $10^{-3}$ corresponds to the target error criterion of both the Gauss Newton and the preconditioned GMRES. We can see that the DD solution exhibits higher levels of errors at the non-regularized edges where the proposed coupling has no effect. It is also possible to see some artifacts across the DD interfaces, but the level of error is around $10^{-4}$ which is reasonably lower than the target error criterion. These artifacts are expected to decrease when the error criterion is lower. Moreover, we compute a global relative error in $L^{2}$-norm 


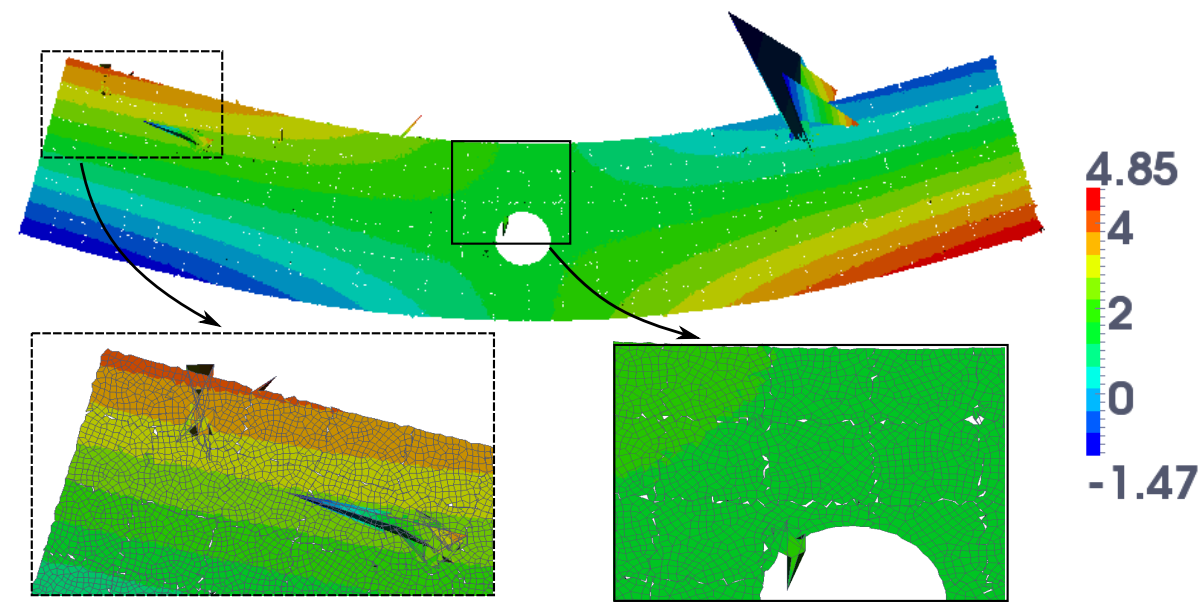

(a) FE-DIC solution (without mechanical regularization) computed independently on each subdomain (block Initialization in Fig. 5).

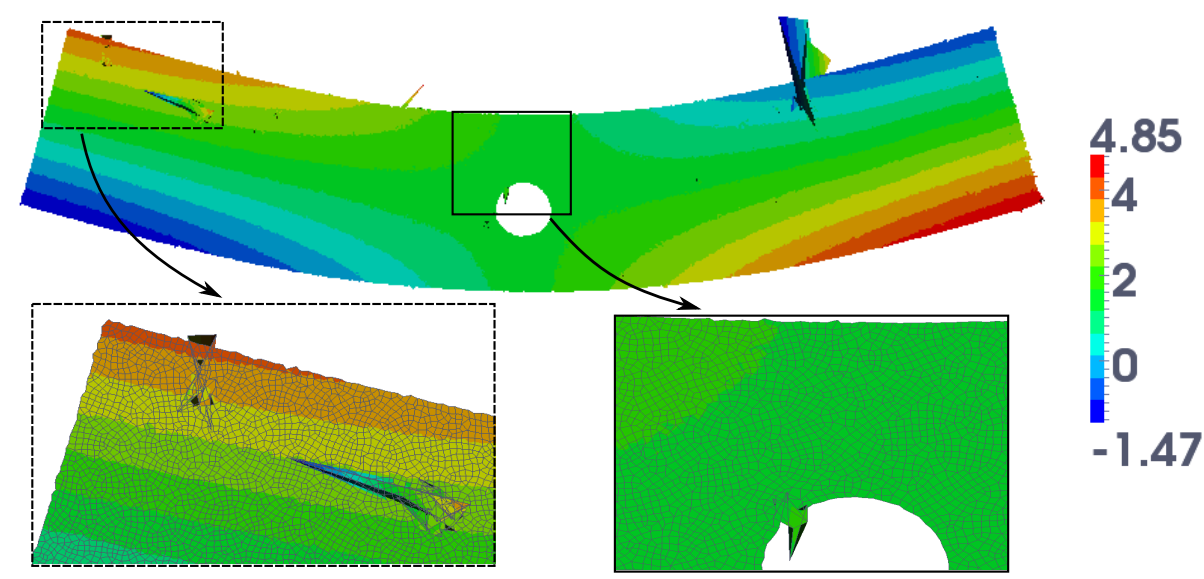

(b) Continuous solution without mechanical regularization obtained with the domain decomposition algorithm of [23].

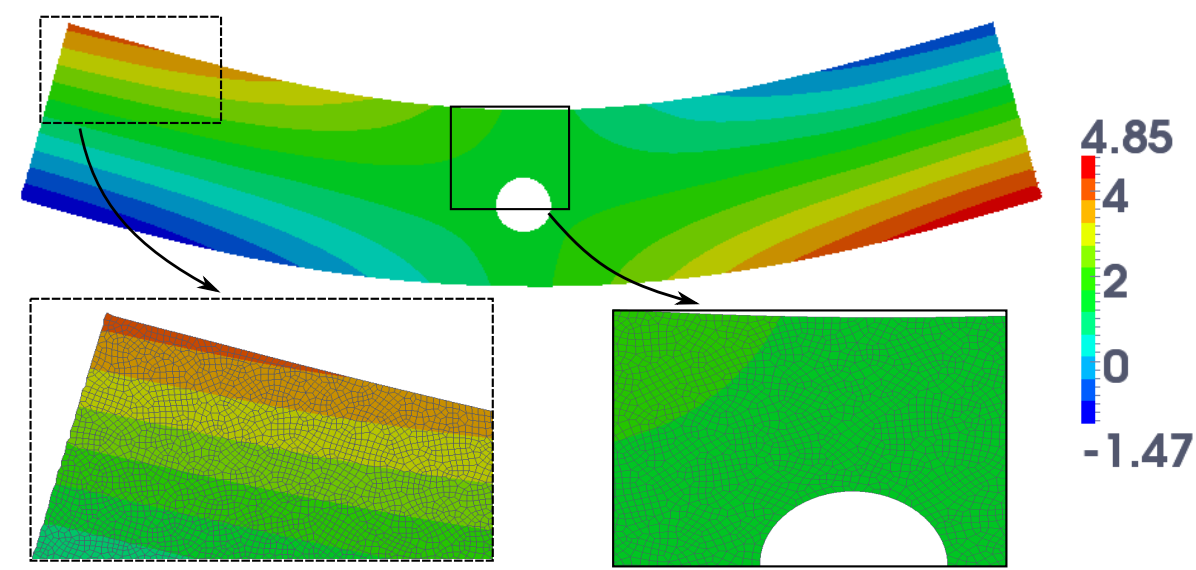

(c) Continuous solution with mechanical regularization obtained with the proposed domain decomposition algorithm (block Correlation iterations in Fig. 5).

Figure 9. Displacement solution in pixels (amplification factor 50, distribution of the horizontal displacement) for the four point bending test. 
between the two displacement fields. Denoting by $\mathbf{q}_{D D}$ and $\mathbf{q}_{\text {mono }}$ the DD and corresponding one-domain d.o.f. vector solution respectively, the global error $\operatorname{Err}$ reads:

$$
E r r=\frac{\left\|\mathbf{q}_{D D}-\mathbf{q}_{\text {mono }}\right\|_{2}}{\left\|\mathbf{q}_{\text {mono }}\right\|}
$$

We obtained a global error of $1.55 \times 10^{-4}$, which again is significantly lower than $10^{-3}$. Such results account for the consistency of the proposed coupling: the obtained DD solution is the same as the corresponding reference one-domain solution.

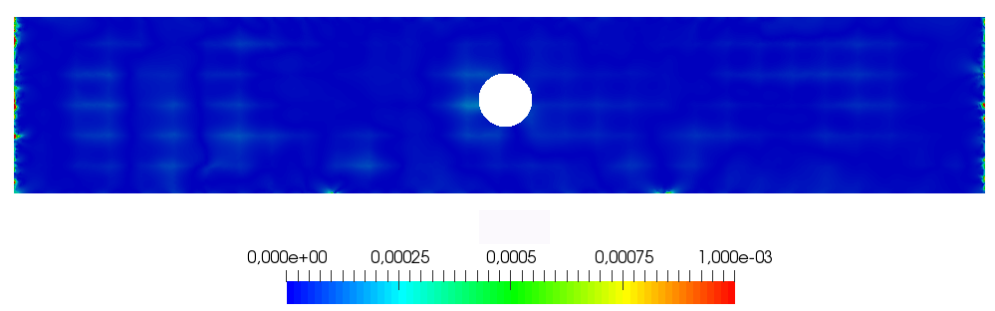

Figure 10. Error between the DD R-DIC $x$-displacement solution and the corresponding reference one-domain R-DIC solution (normalized by the maximum $x$-displacement).

5.3.3. Convergence of the domain decomposition algorithm. We finally investigate the convergence of the domain decomposition algorithm. The variation of the norm of the Krylov residual $\left\|\mathbb{S}_{t o t}^{d} \boldsymbol{\Lambda}_{t o t}^{(j)}-\mathbf{t}_{\text {tot }}^{k}\right\|_{2} /\left\|\mathbf{t}_{t o t}^{k}\right\|_{2}$ (see Alg. 1) as a function of the Krylov iteration $j$ is plotted in Figure 11 for the developed preconditioned GMRES solver (denoted by PGMRES in the figure). For comparison purpose, the solution associated to the GMRES solver without preconditioning (i.e., involving $\mathbb{P}_{i}$ equals to the identity operator) is added to the graph.

These results confirm the efficiency of the parallel initialization of the coupling Gauss-Newton algorithm (see block Initialization in Figure 5). Indeed, it takes in this case only three extra correlation iterations to obtained the desired solution (of Figure 9(c)) from the initialization (shown in Figure 9(a)). It can also be noticed that the number of iterations of the Krylov solver decreases as the correlation iteration $k$ increases, which is due to the use of previous vector $\boldsymbol{\Lambda}_{t o t}$ for the initialization of the Krylov solver. Finally, we observe that the constructed preconditioner allows for a drastic reduction of the number of iterations of the Krylov algorithm (reduction by a factor of ten on this example). With its efficient initialization and preconditioning, the proposed DD algorithm appears then suitable for high performance computing when high resolution images and meshes are jointly used.

Since at this stage only a sequential implementation of the method has been performed in MATLAB, it is not possible to provide a relevant estimate of the speed-up of the DD R-DIC solver with respect to the standard RDIC method. However, providing some implementation cares, it has been possible to observe that one iteration of PGMRES is about $70 \%$ longer than one iteration of GMRES. From a global point of view, taking into account that the number of iterations of the Krylov algorithm is divided by ten with preconditioning, and including the times devoted to the construction and assembling of the preconditioner, we were able to observe that the method involving PGMRES is about 4 times faster than the method using a simple GMRES.

\section{CONCLUSION}

A coupling method was proposed to recover displacement continuity and mechanical equilibrium from two independent finite element digital image correlation analysis with mechanical regularization (R-DIC). Contrary to what is usually done in coupling methods, it was required to introduce an additional interface unknown that is not a Lagrange multiplier for the enforcement of the static constraint. This resulted in the derivation of an original mixed method that has been properly handled to accurately solve the correlation problem. Through a first application, it was shown that such a method could be used to perform the measurement of one smooth and continuous displacement field from two sets of images at nearfield and farfield resolutions respectively. Such a measurement is relevant for the analysis a localized phenomena within large domains. Another application illustrated in the paper 


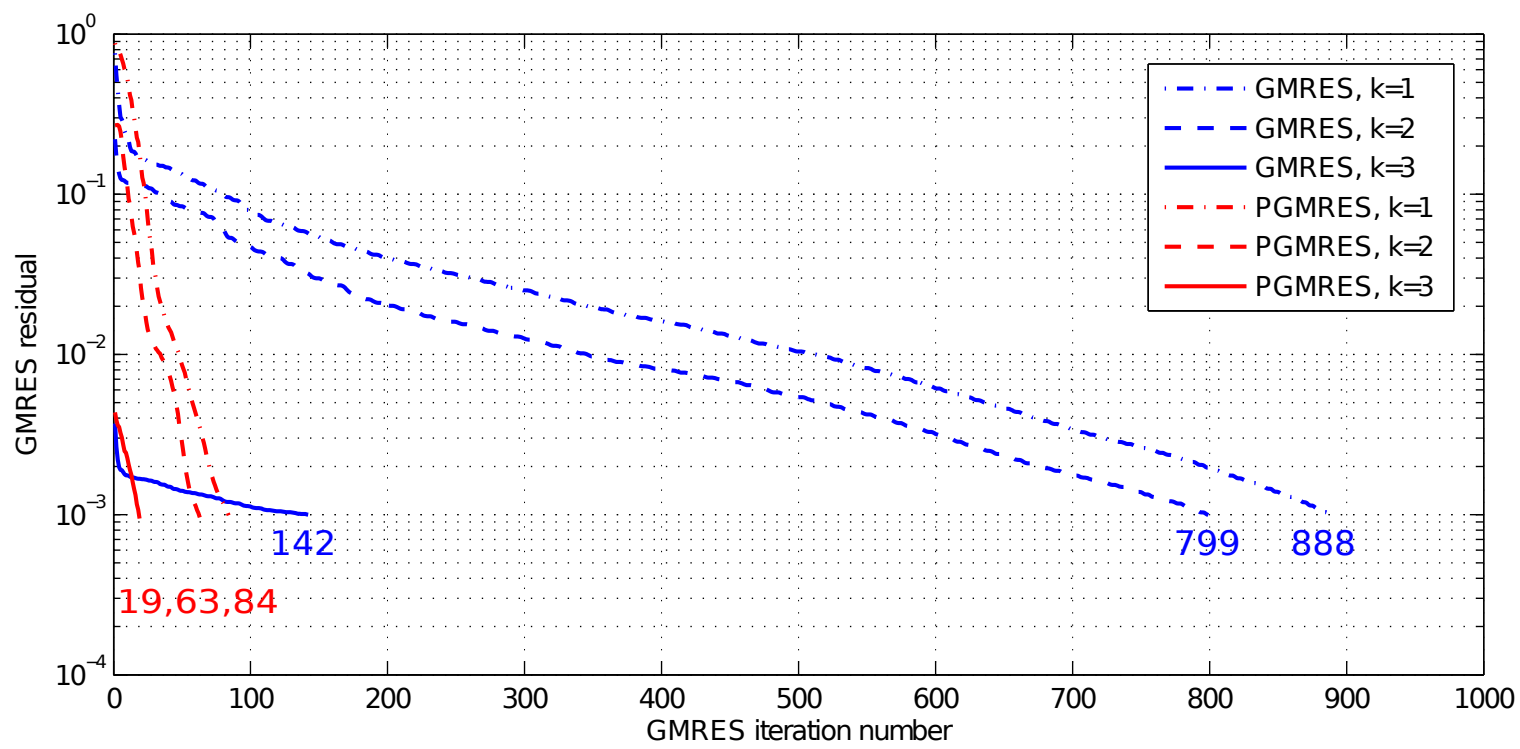

Figure 11. Variation of the norm of the GMRES residual $\left\|\mathbb{S}_{t o t}^{d} \Lambda_{t o t}^{(j)}-\mathbf{t}_{\text {tot }}^{k}\right\|_{2} /\left\|\mathbf{t}_{\text {tot }}^{k}\right\|_{2}$ as a function of the GMRES iteration number $j$ for each correlation iteration $k$ (see block Correlation iterations in Fig. 5). The final number $j$ obtained for each iteration $k$ is reported below the curves. GMRES, GMRES without preconditioning. PGMRES, GMRES with the proposed preconditioner.

is the extension to parallel computing in the context of R-DIC. Since the condensed operator was not positive definite, a GMRES iterative strategy was implemented. In addition, a dedicated preconditioner combining explicit inverses and local primal Schur complements was constructed for acceleration purpose. Such an algorithm allows to benefit from all the great advantages of using FE in DIC (direct bridge with simulation, continuity, mechanical regularization...) while limiting its main drawback, namely computational cost (including computation time and memory requirements). This method may appear useful in a large variety of applications. First, it should constitute a good tool for identification (with the help of the DD identification procedure [45] for example). Then, it would be of great interest for the R-DVC analysis of high resolution volume images. Indeed, todays tomographs commonly provide huge amounts of data that are still out of reach with the most efficient FE DVC approaches [12, 31].

\section{REFERENCES}

[1] Y. Sun, J.H.L. Pang, C. K. Wong, and F. Su. Finite element formulation for a digital image correlation method, Applied Optics, 44 (2005) 7357-7363.

[2] G. Besnard and F. Hild and S. Roux. "Finite-Element" Displacement Fields Analysis from Digital Images: Application to Portevin-Le Châtelier Bands, Experimental Mechanics, 46 (2006) 789-803.

[3] J. Fehrenbach and M. Masmoudi. A fast algorithm for image registration, Comptes Rendus Mathématique, 346 (2008) 593-598.

[4] J. Rannou, N. Limodin, J. Réthoré, A. Gravouil, W. Ludwig, M.-C. Baietto-Dubourg, J.-Y. Buffiére, A. Combescure, F. Hild, and S. Roux. Three dimensional experimental and numerical multiscale analysis of a fatigue crack, Computer Methods in Applied Mechanics and Engineering, 199 (2010) 1307-1325.

[5] R. Fedele, L. Galantucci, A. Ciani, Global 2D digital image correlation for motion estimation in a finite element framework: a variational formulation and a regularized, pyramidal, multi-grid implementation, International Journal for Numerical Methods in Engineering, 96 (2013) 739-762. 
[6] J.-C. Passieux, F. Bugarin, C. David, J.-N. Périé, L. Robert. Multiscale displacement field measurement using digital image correlation: Application to the identification of elastic properties, Experimental Mechanics, 55 (2015) 121-137.

[7] L. Wittevrongel , P. Lava, S. V. Lomov, D. Debruyne. A Self Adaptive Global Digital Image Correlation Algorithm, Experimental Mechanics, 55 (2015) 361-378.

[8] J. Van Beeck, J. Neggers, P.J.G. Schreurs, J.P.M. Hoefnagels, M.G.D. Geers. Quantification of threedimensional surface deformation using global digital image correlation, Experimental Mechanics, 54 (2014) 557-570.

[9] M. Bornert, F. Brémand, P. Doumalin, J.-C. Dupré, M. Fazzini, M. Grédiac, F. Hild, S. Mistou, J. Molimard , J.-J. Orteu, L. Robert, Y. Surrel, P. Vacher and B. Wattrisse. Assessment of Digital Image Correlation measurement errors: methodology and results. Experimental Mechanics 49 (2009) 353-370.

[10] J. Réthoré, S. Roux, F. Hild, An extended and integrated digital image correlation technique applied to the analysis of fractured samples, European Journal of Computational Mechanics, 18 (2009) 285-306.

[11] J. Réthoré. A fully integrated noise robust strategy for the identification of constitutive laws from digital images, International Journal for Numerical Methods in Engineering, 84 (2010) 631-660.

[12] H. Leclerc, J.-N. Périé, S. Roux, and F. Hild. Voxel-scale digital volume correlation. Experimental Mechanics, 51 (2011) 479-490.

[13] H. Leclerc, J.-N. Périé, F. Hild, and S. Roux. Digital volume correlation: What are the limits to the spatial resolution?, Mechanics \& Industry, 13 (2012) 361-371.

[14] J. Réthoré, Muhibullah, T. Elguedj, M. Coret, P. Chaudet, A. Combescure, Robust identification of elastoplastic constitutive law parameters from digital images using 3D kinematics, International Journal of Solids and Structures, 50 (2013) 73-85.

[15] J. Réthoré, Automatic crack tip detection and stress intensity factors estimation of curved cracks from digital images, International Journal for Numerical Methods in Engineering, 103 (2015) 516-534.

[16] Richard B. Lehoucq, Daniel Z. Turner, Carlos A. Garavito-Garz. PDE Constrained Optimization for Digital Image Correlation, Sandia Report Sand2015-8515 (2015).

[17] T.F. Morgeneyer, L. Helfen, H. Mubarak, F. Hild. 3D Digital Volume Correlation of Synchrotron Radiation Laminography images of ductile crack initiation: An initial feasibility study, Experimental Mechanics, 53 (2013) 543-556.

[18] G. Requena, G. Fiedler, B. Seiser, P. Degischer, M. Di Michiel, T. Buslaps. 3D-Quantification of the distribution of continuous fibres in unidirectionally reinforced composites, Composites Part A: Applied Science and Manufacturing, 40 (2009) 152-163.

[19] M. Bornert, F. Valès, H. Garbhi, D. Nguyen Minh. Multiscale Full-Field Strain Measurements for Micromechanical Investigations of the Hydromechanical Behaviour of Clayey Rocks, Strain, 46 (2010) 3346.

[20] J.-C. Passieux, J. Réthoré, A. Gravouil, M.-C. Baietto, Local/global non-intrusive crack propagation simulation using multigrid XFEM solver, Computational Mechanics 52 (2013) 1381-1393.

[21] G. Guguin, O. Allix, P. Gosselet, S. Guinard, Nonintrusive coupling of 3D and 2D laminated composite models based on finite element 3D recovery, International Journal for Numerical Methods in Engineering 98 (2014) 324-343.

[22] R. Bouclier, J.-C. Passieux and M. Salaün, Local enrichment of NURBS patches using a non-intrusive coupling strategy: geometric details, local refinement, inclusion, fracture, Computer Methods in Applied Mechanics and Engineering 300 (2016) 1-26. 
[23] J.-C. Passieux, J.-N. Périé, M. Salaün. A dual domain decomposition method for finite element digital image correlation, International Journal for Numerical Methods in Engineering, 102 (2015) 1670-1682.

[24] B.K.P. Horn, G. Schunck. Determining optical flow, Artificial Intelligence, 17 (1981) 185-203.

[25] S. Roux, J. Réthoré, F. Hild. Digital image correlation and fracture: an advanced technique for estimating stress intensity factors of 2D and 3D cracks, Journal of Physics D: Applied Physics, 42 (2009) 214004.

[26] J. Neggers, B. Blaysat, J. P. M. Hoefnagels, M. G. D. Geers. On image gradients in digital image correlation, International Journal for Numerical Methods in Engineering, 105 (2016) 243-260.

[27] B.D. Lucas, T. Kanade. An iterative image registration technique with an application to stereo vision, In Proceedings of Imaging Understanding Workshop, (1981) 121-130, 1981.

[28] M.A. Sutton, W.J. Wolters, W.H. Peters, W.F. Ranson, and S.R. McNeill. Determination of displacements using an improved digital correlation method, Image and Vision Computing, 1 (1983) 133-139.

[29] M.A. Sutton, J.-J. Orteu, H. Schreier. Image correlation for shape, motion and deformation measurements: Basic Concepts, Theory and Applications, Springer, New York, NY (USA), 2009.

[30] J.-C. Passieux and J.-N. Périé. High resolution digital image correlation using Proper Generalized Decomposition: PGD-DIC, International Journal for Numerical Methods in Engineering, 92 (2012) 531-550.

[31] L.A. Gomes Perini, J.-C. Passieux, and J.-N. Périé. A multigrid PGD-based algorithm for volumetric displacement fields measurements, Strain, 50 (2014) 355-367.

[32] D. Claire, F. Hild, S. Roux. A finite element formulation to identify damage fields: The equilibrium gap method, International Journal for Numerical Methods in Engineering, 61 (2004) 189-208.

[33] F. Hild, S. Roux. Measuring stress intensity factors with a camera: Integrated digital image correlation (IDIC). Comptes Rendus Mécanique, 334 (2006) 8-12.

[34] P. Gosselet, C. Rey, Non-overlapping domain decomposition methods in structural mechanics, Archives of Computational Methods in Engineering, 13 (2006) 515-572.

[35] C. Farhat, F.X. Roux. A method of finite element tearing and interconnecting and its parallel solution algorithm, International Journal for Numerical Methods in Engineering, 32 (1991) 1205-1227.

[36] P. Le Tallec, Y.-H. De Roeck, M. Vidrascu. Domain-decomposition methods for large linearly elliptic three dimensional problems, Journal of Computational and Applied Mathematics, 34 (1991) 93-117.

[37] J. Mandel. Balancing domain decomposition, Communications in Numerical Methods in Engineering, 9 (1993) 233-241.

[38] P. Ladevèze. Nonlinear computationnal structural mechanics-New approaches and non-incremental methods of calculation, Springer Verlag, 1999.

[39] J.-C. Passieux, P. Ladevèze, D. Néron. A scalable time-space multiscale domain decomposition method: adaptive time scale separation, Computational Mechanics, 46 (2010) 621-633.

[40] P. Gosselet, V. Chiaruttini, C. Rey, F. Feyel. A monolithic strategy based on an hybrid domain decomposition method for multiphysic problems. Application to poroelasticity. Revue européenne des élements finis, 13 (2004) 523-534.

[41] Y. Saad. Iterative methods for sparse linear systems, PWS Publishing Company, 3rd edition, 2000.

[42] R. Barrett, M. Berry, T. F. Chan, J. Demmel, J. Donato, J. Dongarra, V. Eijkhout, R. Pozo, C. Romine, H. V. der Vorst. Templates for the Solution of Linear Systems: Building Blocks for Iterative Methods, SIAM, 1994.

[43] Y. Saad and M. H. Schultz. GMRes: a generalized minimal residual algorithm for solving nonsymmetric linear systems, SIAM Journal on Scientific and Statistical Computing, 7 (1986) 856-869. 
[44] P. Gosselet, C. Rey, and J. Pebrel. Total and selective reuse of Krylov subspaces for the resolution of sequences of nonlinear structural problems, International Journal for Numerical Methods in Engineering, 94 (2013) 6083.

[45] A. Moussawi, G. Lubineau, J. Xu, B. Pan. A 3d domain decomposition approach for the identification of spatially varying elastic material parameters, International Journal for Numerical Methods in Engineering, 102 (2015) 1431-1448. 